\title{
Marrying Excitons and Plasmons in Monolayer Transition-Metal Dichalcogenides
}

\author{
Dinh Van Tuan, ${ }^{1, *}$ Benedikt Scharf, ${ }^{2,3,4}$ Igor Žutić, ${ }^{2}$ and Hanan Dery ${ }^{1,5, \dagger}$ \\ ${ }^{1}$ Department of Electrical and Computer Engineering, \\ University of Rochester, Rochester, New York 14627, USA \\ ${ }^{2}$ Department of Physics, University at Buffalo, \\ State University of New York, Buffalo, New York 14260, USA \\ ${ }^{3}$ Institute for Theoretical Physics, University of Regensburg, 93040 Regensburg, Germany \\ ${ }^{4}$ Institute for Theoretical Physics and Astrophysics, \\ University of Würzburg, Am Hubland, 97074 Würzburg, Germany \\ ${ }^{5}$ Department of Physics and Astronomy, University of Rochester, Rochester, New York 14627, USA \\ (Received 14 June 2017; revised manuscript received 7 September 2017; published 17 November 2017) \\ Just as photons are the quanta of light, plasmons are the quanta of orchestrated charge-density \\ oscillations in conducting media. Plasmon phenomena in normal metals, superconductors, and doped \\ semiconductors are often driven by long-wavelength Coulomb interactions. However, in crystals whose \\ Fermi surface is comprised of disconnected pockets in the Brillouin zone, collective electron excitations \\ can also attain a shortwave component when electrons transition between these pockets. In this work, we \\ show that the band structure of monolayer transition-metal dichalcogenides gives rise to an intriguing \\ mechanism through which shortwave plasmons are paired up with excitons. The coupling elucidates the \\ origin for the optical sideband that is observed repeatedly in monolayers of $\mathrm{WSe}_{2}$ and $\mathrm{WS}_{2}$ but not \\ understood. The theory makes it clear why exciton-plasmon coupling has the right conditions to manifest \\ itself distinctly only in the optical spectra of electron-doped tungsten-based monolayers.
}

DOI: 10.1103/PhysRevX.7.041040

\section{INTRODUCTION AND MOTIVATION}

Transition-metal dichalcogenides become direct bandgap semiconductors when thinned to a single atomic monolayer [1,2]. Stacking such two-dimensional crystals via van der Waals forces alleviates the need to fabricate logic and optoelectronic devices with lattice-matched crystals [3-5]. Furthermore, the lack of space inversion symmetry in these monolayers lifts the spin degeneracy at the edges of the conduction and valence bands [6,7]. As a result, time-reversal partners the spin and valley degrees of freedom (d.o.f.) such that spin-up charge carriers populate valleys (low-energy pockets) on one side of the Brillouin zone, while spin-down ones populate valleys on the opposite side [6]. The intense recent research on the spin-valley partnership has led to understanding of optical selection rules and transport phenomena in monolayer transition-metal dichalcogenides (ML-TMDs) [8,9].

In this work, we focus on a unique optical sideband that emerges in emission or absorption-type experiments of electron-doped ML-WSe ${ }_{2}$ and ML-WS ${ }_{2}$ [9-17].

\footnotetext{
*vdinh@ur.rochester.edu

hanan.dery@ rochester.edu
}

Published by the American Physical Society under the terms of the Creative Commons Attribution 4.0 International license. Further distribution of this work must maintain attribution to the author(s) and the published article's title, journal citation, and DOI.
Subject Areas: Condensed Matter Physics

Understanding its microscopic origin has been an outstanding open question ever since its first observation in the photoluminescence (PL) measurements of $\mathrm{ML}^{-\mathrm{WSe}_{2}}$ by Jones et al. [10]. So far, this optical transition has been attributed to the fine structure of negatively charged excitons, biexcitons, or defects in various experiments [9-17]. We show that this optical transition actually corresponds to an exciton-plasmon quasiparticle due to intervalley Coulomb excitations, where other identifications are not consistent with its spectral position, dependence on electron density, and the fact that it does not emerge in hole-doped conditions or in molybdenum-based MLs. To explain the behavior of this optical sideband, we use the empirical data of differential reflectance spectroscopy of $\mathrm{ML}^{-\mathrm{WSe}_{2}}$ at $4 \mathrm{~K}$, as shown in Fig. 1 where this sideband is indicated by $X-^{\prime}$. The monolayer was embedded between thin layers of hexagonal boron nitride in a dual-gated structure [15]. The bias voltage was the same in the top and bottom gate electrodes; thus, only the charge density in the monolayer is controlled, while no out-of-plane electrical field is introduced. The relation between gate voltage and charge density is such that $1 \mathrm{~V}$ corresponds to about $10^{12} \mathrm{~cm}^{-2}$ [15].

Figure 1(a) shows the spectrum for low and intermediate electron densities. The neutral-exciton spectral line, denoted by $X_{0}$, dominates the spectrum, and its intensity decays with increasing the electron density. Also noticeable are three smaller spectral lines in the low-energy side of the spectrum (as highlighted in the enlarged box). These features cannot be completely quenched because of 

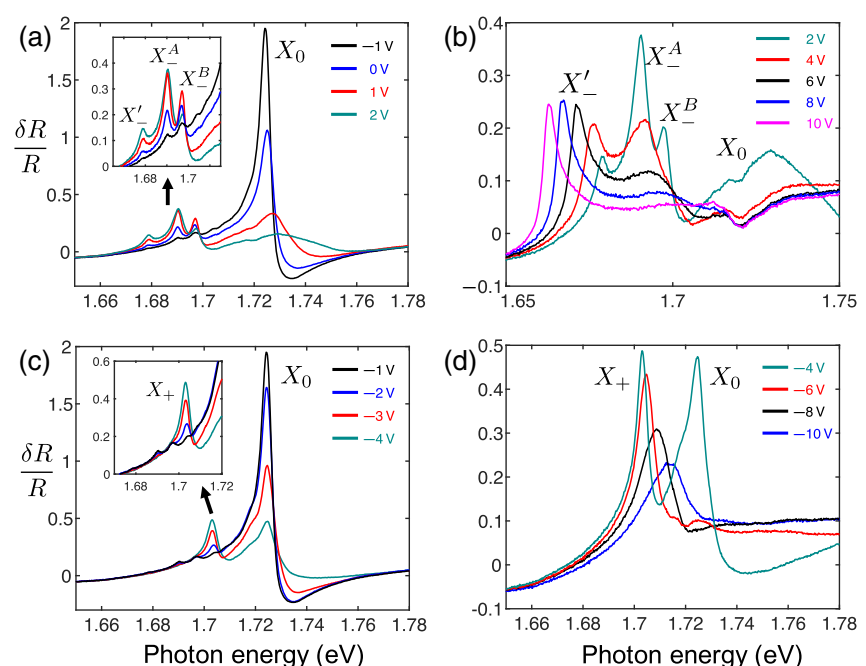

FIG. 1. Optical reflection spectroscopy of $\mathrm{ML}^{-\mathrm{WSe}}{ }_{2}$ at $4 \mathrm{~K}$. The gate voltage controls the charge density in the ML. $(a, b)$ Optical spectra for low and high electron densities, respectively. The optical sideband is indicated by $X-^{\prime}$. (c,d) The respective results for hole densities. The sample is $n$ type, so a gate voltage of about $-1 \mathrm{~V}$ is required to deplete the ML from resident electrons. These results were kindly provided to us by Wang, Mak, and Shan. Reference [15] includes details of the experiment with a similar device.

disordered regions that prevent complete charge depletion across the entire sample [18]. The spectral lines that are denoted by $X_{-}^{A}$ and $X_{-}^{B}$ were recently attributed to two types of negatively charged excitons in tungsten-based monolayers $[14,19,20]$. The lowest-energy spectral line, denoted by $X-^{\prime}$, is the optical sideband. In addition to the fact that $X_{-}^{A}$ and $X_{-}^{B}$ are clearly resolved from that of $X-^{\prime}$ even at low densities, as highlighted in the enlarged box of Fig. 1(a), their behavior is strikingly different, rendering their identification relatively easy. For example, $X_{-}^{A}$ has the known properties of trions in semiconductor quantum wells [21-26]: It is amplified without enhanced broadening when the electron population increases in the low-density regime. In other words, in the regime where the Fermi energy is measurably smaller than the binding energy of the charged exciton (energy difference between $X_{-}^{A}$ and $X_{0}$, which is about $30-35 \mathrm{meV}$ ). Figure 1(b) shows that $X_{-}^{A}$ starts to blueshift, decay, and broaden at intermediate and elevated densities, in which the Fermi energy is no longer negligible compared with the charged-exciton binding energy. In this density regime, the trion cannot avoid many-body interactions with the Fermi sea. The optical sideband, on the other hand, shares none of these features. Figure 1(b) shows that when the electron density continues to increase, $X-^{\prime}$ redshifts and possibly drains part of the oscillator strength of the decaying charged exciton spectral lines. Its spectral line shows no evident broadening, and its saturated intensity at elevated densities hardly decays (whereas the other spectral lines strongly decay, blueshift, and broaden).
Figures 1(c) and 1(d) show the absorption spectrum in hole-doped conditions. It includes the neutral and chargedexciton spectral lines $\left(X_{0}\right.$ and $\left.X_{+}\right)$, where the latter behaves similarly to $X_{-}^{A}$ in terms of its decay, blueshift, and broadening at elevated densities. There is no optical sideband. The reflectivity spectrum of ML-MoSe ${ }_{2}$ is similar (not shown) [15]. It only includes two spectral lines, both in the electron and hole-doping regimes, which behave similarly to $X_{0}$ and $X_{+}$in Figs. 1(c) and 1(d). They share no common properties with the optical sideband of tungsten-based MLs. It is emphasized that in this work we neither model nor focus on the microscopic origin of the charged exciton. Sidler et al. recently suggested that it originates from Fermi polarons, wherein the exciton is attracted to the surrounding Fermi sea via weak van der Waals forces $[27,28]$. Jadczak et al., on the other hand, attributed the charged exciton to trions and further elucidated its strong coupling to phonons due to the resonance between the trion binding energy and that of the homopolar phonon mode [29].

The observed behavior of the optical sideband in tungsten-based compounds is largely identical in reflectance and PL experiments, including the unique feature that the sideband redshifts in both cases $[10,11,15]$. One noticeable difference, however, is the relative amplitude of the signal. Specifically, reflectance measurements show that the largest oscillator strength is that of the neutral exciton at low densities [15,16,20,30,31]. The oscillator strength of the optical sideband, on the other hand, is evidently weaker as one can notice by comparing the $y$-axis scales in Figs. 1(a) and 1(b). The scenario is opposite in PL measurements, where one finds that the strongest light emission comes from the optical sideband at elevated electron densities, while that of the neutral exciton at low densities is evidently weaker $[10,11,15]$. This apparent contradiction is settled in PL experiments for the following reasons: (i) Neutral excitons in tungsten-based monolayers experience intervalley scattering after photoexcitation, rendering them less optically active in the light emission process [32-35]. (ii) The quantum efficiency of light emission can be improved at elevated electron densities because of screening of charged defects that function as nonradiative recombination centers for excitons. (iii) We show that several photon-emission mechanisms contribute to the optical sideband in the PL case.

The main contribution of this work is in elucidating the microscopic origin for the optical sideband, revealing an intriguing pairing phenomenon between excitons and plasmons, which has no parallel in other known twodimensional (2D) systems. We explain why the optical sideband is manifested in the spectra of electron-doped $\mathrm{ML}^{-\mathrm{WSe}_{2}}$ and ML-WS${ }_{2}$ while being absent in the holedoped case or in ML-MoSe 2 and ML-MoS 2 . The theoretical framework in this work studies the oscillator strength of excitons through the electron-hole pair function in the 
presence of dynamical screening. The theory extends beyond the quasistatic random-phase approximation or the Shindo approximation for the dynamical effects [36]. It therefore allows one to model the decay of the neutral exciton at elevated charge densities without introducing phenomenological screening parameters or ansatzes regarding the energy dependence of the pair function. In addition, the theory allows one to model the emergence of dynamical optical sidebands, where we focus on the one that arises from the exciton-plasmon coupling at large electron densities.

\section{BASIC PROPERTIES OF EXCITONS AND PLASMONS IN ML-TMDS}

Before we can explain the coupling between plasmons and excitons, we briefly summarize the relevant properties of each of these quasiparticles separately. Excitons in ML-TMDs are classified based on the spin and valley configuration of the electron-hole pair [32-34]. Figure 2 shows the valleys pertinent to the formation of low-energy excitons in $\mathrm{ML}-\mathrm{WX}_{2}$ and $\mathrm{ML}-\mathrm{MoX}_{2}$, where $\mathrm{X}$ is the chalcogen atom. Excitons are bright or dark (optically active or inactive) if the spins of the electron in the conduction band and the missing electron in the valence band are parallel or antiparallel, respectively. Scattering between dark and bright excitons necessitates a spin-flip of the electron or hole, which is typically a much slower process than the lifetime of bright excitons [37-40]. Therefore, we neglect dark excitons and show that the dynamical relation between two types of bright excitons is sufficient to explain the optical measurements in Fig. 1. The first type is the direct exciton in which the electron and hole have the same valley index, giving rise to direct-gap optical transitions. The second type is the indirect exciton in which the electron and hole reside in opposite valleys. Optical transitions of indirect excitons in perfect crystals are mediated by external agents such as shortwave phonons, which are needed to make up for the large momentum mismatch of indirect excitons and photons. As shown in
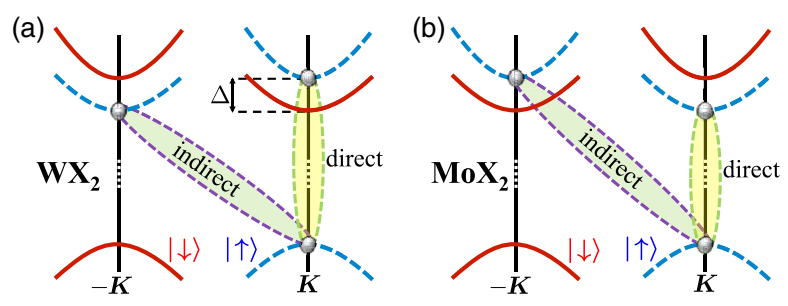

FIG. 2. Direct and indirect excitons in the time-reversed $K$-point valleys. Panels (a) and (b) show the involved electronic states in tungsten- and molybdenum-based monolayers, respectively. The indirect exciton has lower energy in the former case. Spin of the bands is color coded, and $\Delta$ is the conduction-band splitting energy.
Fig. 2, indirect excitons have lower energy than direct ones in $\mathrm{ML}^{-\mathrm{WX}_{2}}$ and vice versa in ML-MoX $\mathrm{M}_{2}$ [32].

Next, we briefly discuss the two plasmon species in ML-TMDs. The first type of plasmon is characterized by its long wavelength [41-43]. These plasmons originate from the long-range part of the Coulomb potential through which electrons cross the Fermi surface with small crystal momentum transfer (i.e., an intravalley process). They are common to all conducting media and are largely independent of the band structure since their wavelength is much longer than the lattice constant [44]. In the context of optical transitions in semiconductors, plasmons screen the electron-hole attraction, and they shrink the band-gap energy by assisting electrons (or holes) of similar spins to avoid each other [36]. The energy dispersion of longwavelength plasmons in 2D semiconductors follows,

$$
E_{\ell}(k)=\sqrt{\frac{2 e^{2} E_{F} k}{\epsilon(k)}},
$$

where $e$ is the elementary charge, $k$ is the wave number of the plasmon, and $E_{F}=\pi \hbar^{2} n / m_{e}$ is the Fermi energy related to the charge density and effective mass of the electron $\left(n\right.$ and $\left.m_{e}\right)$. The static dielectric function $\epsilon(k)$ takes into account the experimental sample geometry, as depicted in Fig. 3. Its form follows [45],

$$
\frac{1}{\epsilon(k)}=\frac{1}{\epsilon_{\mathrm{ML}}} \cdot \frac{1+\left(p_{b}+p_{t}\right) e^{-k d}+p_{b} p_{t} e^{-2 k d}}{1-p_{b} p_{t} e^{-2 k d}}
$$

where $d$ denotes the thickness of the semiconductor, including the van der Waals gap, and $\epsilon_{\mathrm{ML}}$ is the dielectric constant of the ML. Here, $p_{b}\left(p_{t}\right)$ is the polarization due to the dielectric contrast between the ML and the bottom (top) layer,

$$
p_{b(t)}=\frac{\epsilon_{\mathrm{ML}}-\epsilon_{b(t)}}{\epsilon_{\mathrm{ML}}+\epsilon_{b(t)}}
$$

where $\epsilon_{b}\left(\epsilon_{t}\right)$ is the dielectric constant of the bottom (top) layer. In the long-wavelength regime, one can readily show that the effective dielectric constant is largely set by the

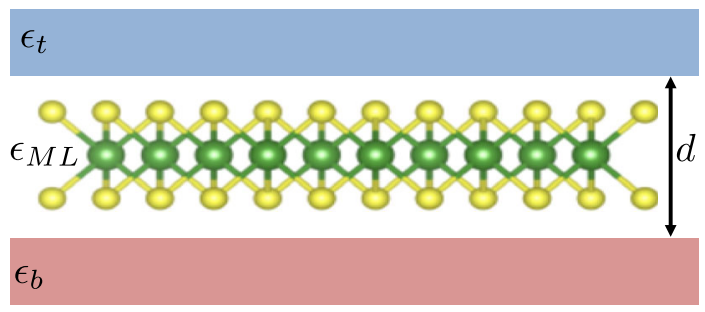

FIG. 3. The geometry studied in this work is that of a ML-TMD sandwiched between two other materials. The thickness of the ML, including the van der Waals gap, is $d$. The dielectric constants of the ML, bottom, and top layers are $\epsilon_{\mathrm{ML}}, \epsilon_{b}$, and $\epsilon_{t}$, respectively. 
average dielectric constants of the top and bottom layers $[45,46]$,

$$
\epsilon(k) \stackrel{k d \ll 1}{\longrightarrow} \frac{\epsilon_{t}+\epsilon_{b}}{2}+\left(\epsilon_{\mathrm{ML}}-\frac{\epsilon_{t}^{2}+\epsilon_{b}^{2}}{2 \epsilon_{\mathrm{ML}}}\right) \frac{k d}{2} .
$$

The second type of plasmon originates from the shortrange Coulomb potential through which electrons transition between valleys [35,47-50]. Because of the relatively large spin splitting in the valence band of ML-TMDs [6], such transitions are mostly relevant in the conduction band wherein the much smaller spin splitting can be comparable to the Fermi energy, as shown in Fig. 4(a). Collective intervalley excitations result in shortwave plasmons, whose charge-fluctuations profile is illustrated in Fig. 4(b), and whose energy dispersion follows [35]

$$
E_{\mathrm{s}}(q)=\Delta+\left(1+\frac{3}{\alpha}\right) \tilde{\varepsilon}_{q}+\frac{\alpha}{3} E_{F} .
$$

Note that $\Delta$ is the spin-splitting energy in the conduction band, $\alpha=\left(K_{0} a_{\mathrm{s}}\right)^{-1} \sim 0.1$, and $\tilde{\varepsilon}_{q}=\hbar^{2}\left(K_{0}-q\right)^{2} / 2 m_{e}$. Here, $K_{0}=4 \pi / 3 a$ is the wave number that connects the time-reversed valleys $(a \sim 3.2 \AA$ is the lattice constant), and $a_{\mathrm{s}}=\hbar^{2} \epsilon_{\mathrm{ML}} / e^{2} m_{e}$ is the effective Bohr radius at these short wavelengths. Microscopic corrections due to the short-range Coulomb potential can be incorporated in Eq. (5) via a change in the value of $\alpha$. The reason for this is that the dielectric constant loses its meaning when $k d>1$ if the thickness of the ML is comparable to the lattice constant [46]. Courtade et al. have used empirical values of the fine structure of charged excitons and estimated that the amplitude of the short-range Coulomb potential is of the order of a few $\mathrm{eV} \cdot \AA^{2}$ [20]. This

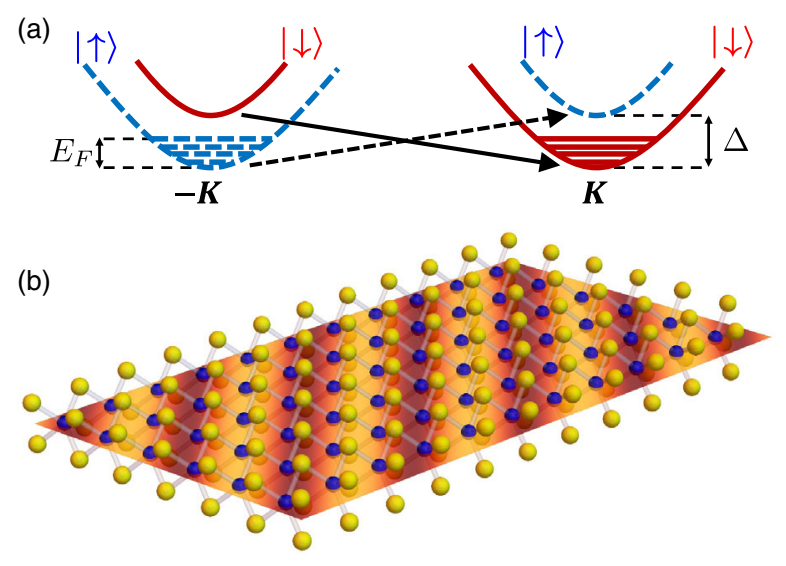

FIG. 4. The intervalley Coulomb interaction in ML-TMDs. (a) Spin-conserving charge excitations from the $-K$ to the $K$ valleys. Electrons need to overcome the spin-splitting energy gap in the conduction band. (b) The resulting shortwave charge fluctuations in the monolayer. amplitude is similar to the value of the intervalley Coulomb potential, $2 \pi e^{2} / \epsilon_{\mathrm{ML}} K_{0}$, that was used to derive Eq. (5), indicating that $\alpha \sim 0.1$ remains a good estimate even when microscopic corrections are taken into account. Note that the existence of short-wave plasmons only depends on the fact that the short-range Coulomb potential is not negligible, regardless of its exact value. The energy dispersion in Eq. (5) is derived by assuming that the shortrange Coulomb potential hardly changes when $k_{F} \ll K_{0}$, where $k_{F}$ is the Fermi wave number. Indeed, $k_{F}$ is more than 1 order of magnitude smaller than $K_{0}$ even when the density is of the order of $10^{13} \mathrm{~cm}^{-2}$. The region of freeplasmon propagation is then limited to $K_{0}-(\alpha / 3) k_{F}<$ $q<K_{0}+(\alpha / 3) k_{F}$ [35]. In other words, when the wave number of a shortwave plasmon belongs to a small region of nearly perfect time-reversal intervalley conditions $\left(q \sim K_{0}\right)$, it does not interact with individual electrons and should be treated as an independent degree of freedom in the electronic system [44].

In addition to their different length scales, intravalley and intervalley plasmons in ML-TMDs differ in their energy spectrum. The spin splitting in the conduction band produces a gapped dispersion for shortwave plasmons compared with the gapless dispersion in the longwavelength case: $E_{\mathrm{s}}\left(K_{0}\right)=\Delta+\alpha E_{F} / 3$ while $E_{\ell}(0)=0$. The value of $\Delta$ is governed by the spin-orbit coupling at vanishing electron densities. Its value increases at elevated densities because of the electron-electron exchange interaction [35].

\section{PAIR FUNCTION OF EXCITONS IN THE PRESENCE OF PLASMONS}

To study how excitons interact with plasmons, we use the finite-temperature Green's functions formalism [51] and quantify the optical signature of bright excitons when surrounded by collective charge excitations [36]. The connection to the absorption spectrum is then established via the imaginary part of the electron-hole pair function,

$A(\omega) \propto \beta^{-1} \sum_{\mathbf{k}, z} \operatorname{Im}\left\{G_{p}\left(\mathbf{q} \rightarrow 0, \mathbf{k}, z, \Omega \rightarrow E_{\omega}+i \delta\right)\right\}$.

Here, $\hbar \omega$ is the photon energy and $\beta^{-1}=k_{B} T$ is the thermal energy. Note that $\mathbf{k}$ and $\mathbf{q}$ are $2 \mathrm{D}$ crystal wave vectors, where the electron in the pair takes on $\mathbf{k}+\mathbf{q}$ and the hole $-\mathbf{k}$. In the limit where $\mathbf{q} \rightarrow 0$, a direct optical transition is rendered because of the small momentum of photons. Throughout the analysis below, pair functions of direct and indirect excitons are evaluated by assigning $q=0$ and $q=K_{0}$, respectively. Dynamical effects due to plasma oscillations are embodied by the dependence of $G_{p}$ on $z$ and $\Omega$, which are odd (fermion) and even (boson) imaginary Matsubara energies, $z=(2 \ell+1) \pi i / \beta$ and $\Omega=2 \ell \pi i / \beta$, where $\ell$ is an integer [36]. The sums over $\mathbf{k}$ and $z$ in Eq. (6) integrate out the fermion d.o.f. In the last 


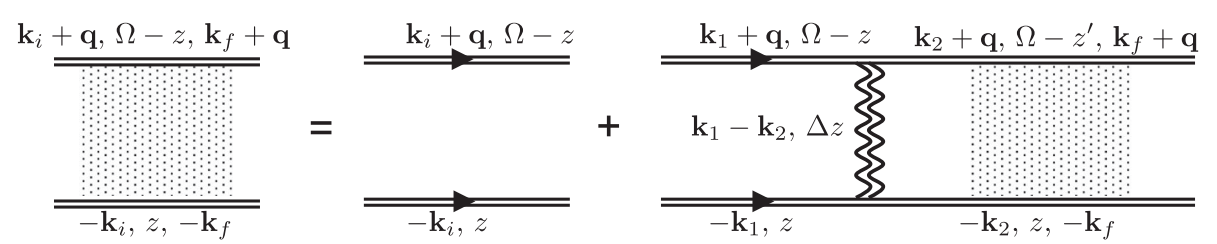

FIG. 5. Feynman diagram representation of the two-particle Dyson equation under the screened ladder approximation. The top and bottom propagators denote the Green's functions of electrons and holes, respectively. The wiggly line denotes the dynamically screened attractive potential. This diagram sums over all processes through which the electron-hole pair is repeatedly scattered by the attractive potential.

step, Padé analytical continuation is performed in order to evaluate the pair function at real photon energies [52]: $\Omega \rightarrow E_{\omega}+i \delta$, where $\delta \rightarrow 0^{+}$and $E_{\omega}=\hbar \omega-\mu_{e}-\mu_{h}$ is expressed in terms of photon energy and quasichemical potentials in the conduction and valence bands.

To find the pair function in Eq. (6), we make use of the so-called screened ladder approximation. It describes repeated interaction of the electron-hole pair with the dynamically screened potential [36]. While this physical picture considers the interaction of excitons with collective electron excitations, it neglects exciton-exciton interactions. Accordingly, the screened ladder approximation can be used to explain experiments in which the ML is not subjected to intense photoexcitation. Figure 5 shows the Feynman diagram of the two-particle Green's function before contraction (i.e., before integrating out any of its quantum numbers). This diagram is formally written as

$$
G_{p}\left(\mathbf{q}, \mathbf{k}_{i}, \mathbf{k}_{f}, z, \Omega\right)=G_{p, 0}\left(\mathbf{q}, \mathbf{k}_{i}, \mathbf{k}_{f}, z, \Omega\right)+\frac{1}{\beta} \sum_{\mathbf{k}_{1}, \mathbf{k}_{2}, z^{\prime}} G_{p, 0}\left(\mathbf{q}, \mathbf{k}_{i}, \mathbf{k}_{1}, z, \Omega\right) V_{s}\left(\mathbf{k}_{1}-\mathbf{k}_{2}, z-z^{\prime}\right) G_{p}\left(\mathbf{q}, \mathbf{k}_{2}, \mathbf{k}_{f}, z^{\prime}, \Omega\right),
$$

where $V_{s}\left(\mathbf{k}-\mathbf{k}^{\prime}, z-z^{\prime}\right)$ is the dynamically screened potential to be discussed later. Here, $G_{p}\left(\mathbf{q}, \mathbf{k}_{i}, \mathbf{k}_{f}, z, \Omega\right)$ and $G_{p, 0}\left(\mathbf{q}, \mathbf{k}_{i}, \mathbf{k}_{f}, z, \Omega\right)$ are the interacting and noninteracting pair functions, respectively. The noninteracting case is given by the product of the electron and hole Green's functions

$G_{p, 0}\left(\mathbf{q}, \mathbf{k}_{i}, \mathbf{k}_{f}, z, \Omega\right)=G_{e}\left(\mathbf{k}_{i}+\mathbf{q}, \Omega-z\right) G_{h}\left(-\mathbf{k}_{i}, z\right) \delta_{\mathbf{k}_{i}, \mathbf{k}_{f}}$,

where here and below we assume the area of the monolayer is 1 . The Kronecker delta function allows us to integrate out the final wave vector $\mathbf{k}_{f}$ in Eq. (7) and to calculate the contracted pair function $G_{p}(\mathbf{q}, \mathbf{k}, z, \Omega)$, from which we can estimate the absorption according to Eq. (6). We note that while this step seems trivial, the approach so far was to contract over the odd Matsubara energy $(z)$ and then use the so-called Shindo approximation to describe dynamical screening [36,53]. However, dynamical screening can be better described upon contraction in momentum rather than energy space. This contraction keeps both dynamical parameters (even and odd Matsubara energies), while eliminating $\mathbf{k}_{f}$ does not compromise any further accuracy in the solution of the pair function if the starting point is the screened ladder approximation. After contraction, we simplify the form of Eq. (7),

$$
G_{p}(\mathbf{q}, \mathbf{k}, z, \Omega)=G_{e}(\mathbf{k}+\mathbf{q}, \Omega-z) G_{h}(-\mathbf{k}, z)\left\{1+\beta^{-1} \sum_{\mathbf{k}^{\prime}, z^{\prime}} V_{s}\left(\mathbf{k}-\mathbf{k}^{\prime}, z-z^{\prime}\right) G_{p}\left(\mathbf{q}, \mathbf{k}^{\prime}, z^{\prime}, \Omega\right)\right\} .
$$

This equation can be solved by matrix inversion techniques, as explained in the Appendix.

\section{A. Effect of long-wavelength plasmons on excitons}

We begin by evaluating the pair functions of direct excitons when $V_{s}, G_{e}$, and $G_{h}$ in Eq. (9) are affected only by long-wavelength plasmons. Neglecting shortwave plasmons in the first step allows us to find common attributes between ML-TMDs and conventional semiconductor quantum wells [54].

A central part of the theory is the inclusion of the dynamically screened potential in Eq. (9). We invoke the single-plasmon pole approximation (SPP), which provides a relatively compact form for the dynamical screening. Using the more rigorous random-phase approximation does not lead to qualitative changes or to increased 
computational complexity when solving Eq. (9). When including long-wavelength plasma excitations, the dynamically screened Coulomb potential under the SPP approximation reads [36]

$$
V_{s}\left(\mathbf{k}, z-z^{\prime}\right)=\frac{2 \pi e^{2}}{\epsilon(k)} \frac{1}{k}\left(1+\frac{E_{\ell}^{2}(k)}{\left(z-z^{\prime}\right)^{2}-E_{k}^{2}}\right),
$$

where

$$
E_{k}=E_{\ell}(k) \sqrt{1+\frac{a_{\ell} k}{4}}
$$

is the pole energy, and $a_{\ell}=\hbar^{2}\left(\epsilon_{b}+\epsilon_{t}\right) / 2 e^{2} m_{e}$ is the effective Bohr radius at long wavelengths. The solution of Eq. (9) is greatly simplified when using the static approximation $V_{s}(\mathbf{k}, 0)$ or when studying the zero density case $E_{\ell}(k)=0$. In these cases, one first calculates the sum $\sum_{z} G_{e}(\mathbf{k}+\mathbf{q}, \Omega-z) G_{h}(-\mathbf{k}, z)$ and then directly calculates the absorption spectrum from the contracted pair function $G_{p}(\mathbf{q}, \mathbf{k}, \Omega)$. The static approximation, however, tends to overestimate the role of screening, and one has to introduce phenomenological terms in the static potential to mitigate the screening effect [54,55]. A better description for the screening is provided by keeping the dynamical terms in the potential, $V_{s}\left(\mathbf{k}, z-z^{\prime}\right)$. This approach, however, renders the solution of Eq. (9) evidently more computationally demanding compared with the static case.

The second signature of long-wavelength plasmons is introducing the band-gap renormalization (BGR) through the self-energy terms in the electron and hole Green's functions,

$$
G_{i}(\mathbf{k}, z)=\frac{1}{z-\varepsilon_{\mathbf{k}, i}-\Sigma_{i}(\mathbf{k}, z)+\mu_{i}},
$$

where $i=\{e, h\}$, and $\varepsilon_{\mathbf{k}, i}=\hbar^{2} k^{2} / 2 m_{i}$ is the noninteracting electron (or hole) energy. The self-energy term $\Sigma_{i}(\mathbf{k}, z)$ stems from the interaction of the electron (or hole) with the dynamically screened potential, and $\mu_{i}=\mu_{i, 0}+$ $\Sigma_{i}\left(\mathbf{k}_{F}, E_{F}-\mu_{i, 0}\right)$ is the chemical potential. Here, $\mu_{i, 0}$ is the temperature- and density-dependent chemical potential of the noninteracting electron gas. Using the $G W$ approximation, the self-energy term is written as [36]

$$
\begin{aligned}
\Sigma_{i}(\mathbf{k}, z) & =-\beta^{-1} \sum_{\mathbf{q}, \Omega} V_{s}(\mathbf{q}, \Omega) G_{i, 0}(\mathbf{k}-\mathbf{q}, z-\Omega) \\
& =\Sigma_{x, i}(\mathbf{k})+\Sigma_{c, i}(\mathbf{k}, z),
\end{aligned}
$$

where $G_{i, 0}$ is the Green's function of the noninteracting particle $(\Sigma=0)$. The self-energy is separated into exchange and correlation parts. The former follows

$$
\Sigma_{x, i}(\mathbf{k})=-\sum_{\mathbf{q}} \frac{2 \pi e^{2}}{\epsilon(q)} \frac{f_{i}(\mathbf{k}-\mathbf{q})}{q},
$$

where $f_{i}$ is the Fermi-Dirac distribution. The exchange contribution from the electron-electron interaction can only shift the filled valleys (i.e., $f_{i} \neq 0$ ). The correlation term, on the other hand, affects all bands, and it comes from emission or absorption of long-wavelength plasmons (intravalley processes),

$$
\begin{aligned}
\Sigma_{c, i}(\mathbf{k}, z)= & \sum_{\mathbf{q}<q_{c}} \frac{2 \pi e^{2}}{\epsilon(q)} \frac{1}{q} \frac{E_{\ell}^{2}(q)}{2 E_{q}} \\
& \times\left\{\frac{f_{i}(\mathbf{k}-\mathbf{q})+g\left(E_{q}\right)}{z-\varepsilon_{\mathbf{k}-\mathbf{q}, i}+\mu_{i}+E_{q}}-\left(E_{q} \leftrightarrow-E_{q}\right)\right\},
\end{aligned}
$$

where $g\left(E_{q}\right)$ is the Bose-Einstein distribution function evaluated at the plasmon pole energy, and $q_{c}$ is the integration cutoff introduced to guarantee convergence. Physically, it amounts to avoiding plasma damping due to particle-hole excitations of the Fermi sea.

We now have all of the ingredients to solve the pair function in Eq. (9) and to then calculate the absorption spectrum from Eq. (6) after performing analytical continuation from the imaginary to the real axis (see the Appendix for more details). Figure 6 shows the calculated absorption spectrum at $T=10 \mathrm{~K}$ from which one can clearly notice

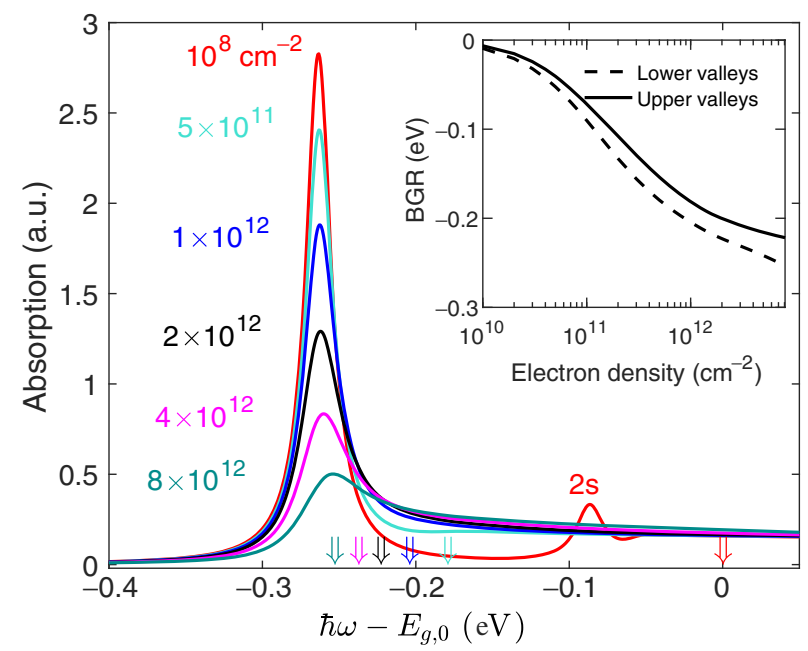

FIG. 6. Characteristic effect of long-wavelength plasmons on the absorption spectrum of neutral excitons in ML-TMDs. Increasing the background charge density leads to a decay of the exciton peak. The BGR is indicated by the arrows on the $x$ axis, showing the redshift for the onset of optical transitions in the continuum (noninteracting electron-hole pairs). Inset: BGR of the upper and lower valleys as a function of electron density in the lower valleys. The signatures of dynamical screening and BGR are qualitatively the same when choosing other parameters of effective masses and dielectric constants (Appendix A 5). 
the exciton decay when the background charge density increases. The effective mass for both electrons and holes is $0.36 m_{0}$ [56], where $m_{0}$ is the free-electron mass (see Appendix A 1). The dielectric environment is modeled by sandwiching the ML $\left(d=1 \mathrm{~nm}, \epsilon_{\mathrm{ML}}=7.25\right)$ between thick layers of hexagonal boron nitride $\left(\epsilon_{b}=\epsilon_{t}=2.7\right)$ [15,57]. The arrows in Fig. 6 indicate the continuum redshift due to BGR. Above this energy, the excitons are no longer bound. Note that the $2 s$ bound state merges into the continuum already at relatively small densities because of its smaller binding energy. The BGR is calculated from the value of $\Sigma_{i}\left(\mathbf{k}, \varepsilon_{\mathbf{k}, i}-\mu_{i}^{0}\right)$ at $\mathbf{k}=0$, by assuming a rigid shift of the bands. The cutoff energies we have used for the bottom and top spin-split valleys in Eq. (15) are $\varepsilon_{q_{c}}=$ $60 \mathrm{meV}$ and $\varepsilon_{q_{c}}=45 \mathrm{meV}$, respectively. These cutoff energies are the same for all densities. Using larger (smaller) cutoff energies, the peak position will gradually redshift (blueshift) as one increases the charge density. The inset of Fig. 6 shows that the BGR is strongest when the charge density is ramped up from zero to about $10^{12} \mathrm{~cm}^{-2}$. The continuum redshift is much slower between about $10^{12}$ and $10^{13} \mathrm{~cm}^{-2}$, during which excitons become unbound and merge into the continuum.

Two effects cause the decay of the neutral-exciton spectral line with the increase in electron density. The dominant one is the broadening effect when the exciton energy approaches the continuum [see arrows in Fig. 6 and Eq. (A2) in the Appendix]. The decay that is caused by reduction in the oscillator strength plays a secondary role due to the mitigated effect of screening in 2D. This behavior is reminiscent of excitonic enhancement of free electron-hole transitions in semiconductor quantum wells [55], in which the overlap between the electron and hole wave functions remains sizable when excitons enter the continuum. The simulated decay of the neutral exciton in Fig. 6 is corroborated in the differential-reflectivity measurements, which resolve the oscillator strength and broadening of the spectral lines [15,16]. Figures 1(a) and 1(c) show that when the charge density is ramped up by gate voltage, the measured decay of neutral excitons is not compensated by an equivalent increase of charged excitons, thereby reinforcing the important role of BGR and dynamical screening. It is noted that, unlike the experimental findings of Fig. 1, the simulated exciton peak in Fig. 6 is not completely quenched at elevated densities. In addition to the fact that we do not consider charged excitons, which drain part of the oscillator strength of neutral excitons [58], the model tends to overestimate the Coulomb attraction at elevated densities. The reason is that broadening effects are not introduced in the electron and hole Green's functions of Eq. (9); they are introduced in the analytical continuation phase after the pair function is solved (in the Appendix).

An additional important result in Fig. 6, which is supported by Figs. 1(a) and 1(c), is that the neutral exciton spectral position is hardly affected by the background charge density because of the mutual offset between BGR and the screening-induced reduction in the binding energy of excitons; the long-wavelength plasmons are responsible for both effects [54]. One can therefore realize that the binding energy of excitons in ML-TMDs can be of the order of a few hundred meV only at vanishing charge densities. All in all, long-wavelength plasmons lead to qualitatively similar effects in ML-TMDs and conventional semiconductor quantum wells [55]. The only change is a quantitatively larger effect in ML-TMDs due to reduced dielectric screening and larger electron mass $[59,60]$.

\section{B. Effect of shortwave plasmons on excitons}

In the next step, the unique features of ML-TMDs are revealed by turning on the coupling between shortwave plasmons and excitons. In the long-wavelength case, the exciton-plasmon coupling is implicit: It is embodied in the self-energies of the electron and hole as well as in the attractive screened potential (i.e., the coupling to plasmons is "concealed" in the dressed propagator and Coulomb lines in Fig. 5). An explicit exciton-plasmon interaction is inhibited if the extension of the exciton is smaller than the wavelength of the plasmon because of the charge neutrality of the former. This physical picture changes for shortwave plasmons, which can be effectively paired up with an exciton in spite of its charge neutrality. Here, the short-range nature of the charge fluctuations, as can be seen (a)

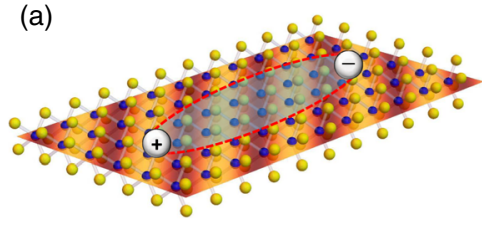

(b)

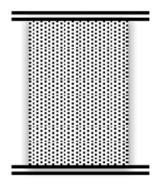

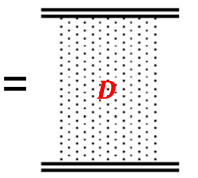

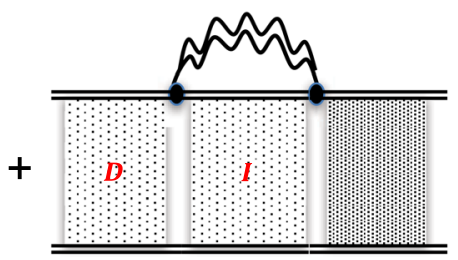

FIG. 7. Coupling between excitons and shortwave plasmons. (a) Cartoon of the exciton and shortwave charge fluctuations. The plasmon wavelength is shorter than the exciton extent, which is further blown up because of screening. (b) Feynman diagram representation of the renormalized direct-exciton pair function due to shortwave plasmons. The self-energy term takes into account the coupling between direct and indirect excitons ( $D$ and $I$ ) due to the electron-plasmon interaction. The hole (bottom propagator) acts as a spectator, while the electron interacts with the plasmon. The double wiggly lines in this diagram denote the plasmon propagator, and the vertices denote the electronplasmon interaction. The diagram is an infinite sum of the virtual processes $D+D I D+D I D I D+\cdots$. 
in Fig. 7(a), allows the hole or electron to act as a spectator while the plasmon interacts with the opposite charge.

The energy required to excite a shortwave plasmon at low temperatures is greater than that available thermally $\left(\Delta \gg k_{B} T\right)$. In addition, we recall that free-propagating plasmons are independent d.o.f., which do not interact with individual charged particles of the electronic system. As a result, plasmon signatures in the optical spectrum can be resolved by supplying energy from outside the electron system in amounts greater than the plasmon energy. This is the case when we shake up the electronic system by exciting electron-hole pairs. Figure 7(b) shows the Feynman diagram for the explicit coupling between an exciton and a shortwave plasmon when the hole acts as the spectator. The plasmon propagator is the double wiggly line. This Feynman diagram corresponds to renormalization of the pair function of direct excitons according to

$$
\tilde{G}_{p}(\mathbf{q} \rightarrow 0, \mathbf{k}, \Omega)=\frac{G_{p}(\mathbf{q} \rightarrow 0, \mathbf{k}, \Omega)}{1-\Sigma_{s}(\mathbf{k}, \Omega) G_{p}(\mathbf{q} \rightarrow 0, \mathbf{k}, \Omega)},
$$

where the pair Green's functions were contracted by integrating out the fermion Matsubara energies. The mixing with indirect excitons comes from the self-energy term,

$$
\Sigma_{s}(\mathbf{k}, \Omega)=\beta^{-1} \sum_{\mathbf{q}^{\prime}, \Omega^{\prime}} M_{\mathbf{q}^{\prime}}^{2} D\left(\Omega-\Omega^{\prime}, \mathbf{q}^{\prime}\right) G_{p}\left(\mathbf{q}^{\prime}, \mathbf{k}, \Omega^{\prime}\right),
$$

where $M_{\mathbf{q}^{\prime}}$ is the interaction between the plasmon and the electron component of the exciton, and $D(\Omega, \mathbf{q})$ is the freeplasmon propagator. The sum in Eq. (17) is restricted to intervalley transitions, $q^{\prime} \rightarrow K_{0}$, so that the pair function on the right-hand side is that of indirect excitons. The interaction and propagator terms follow [44],

$$
\begin{aligned}
& M_{\mathbf{q}^{\prime}}=\frac{\sqrt{3 \pi} \hbar^{2}\left|\mathbf{K}_{0}-\mathbf{q}^{\prime}\right|}{m_{e}}, \\
& D(\Omega, \mathbf{q})=\frac{2 E_{\mathrm{s}}(q)}{\Omega^{2}-E_{\mathrm{s}}^{2}(q)} .
\end{aligned}
$$

Figure 8 shows the renormalized absorption spectrum of direct excitons in electron-doped ML-WX $\mathrm{W}_{2}$ at $10 \mathrm{~K}$. A dynamical sideband emerges in the low-energy side of the spectrum. We have considered tungsten-based settings such that indirect excitons have lower energy than direct ones [Fig. 2(a)]. The spin-orbit coupling splits the conductionband valleys by $26 \mathrm{meV}$ [61]. All other parameters are the same as in Fig. 6. The behavior of the simulated dynamical sideband is similar to the empirical behavior of the optical sideband in electron-doped ML-WX $\mathrm{W}_{2}$ [9-16], as shown in Fig. 1(b). Both the theoretical and the experimental results show a redshift and amplification of the optical sideband when increasing the electron density until they saturate. We note that the pair function reflects the oscillator strength of

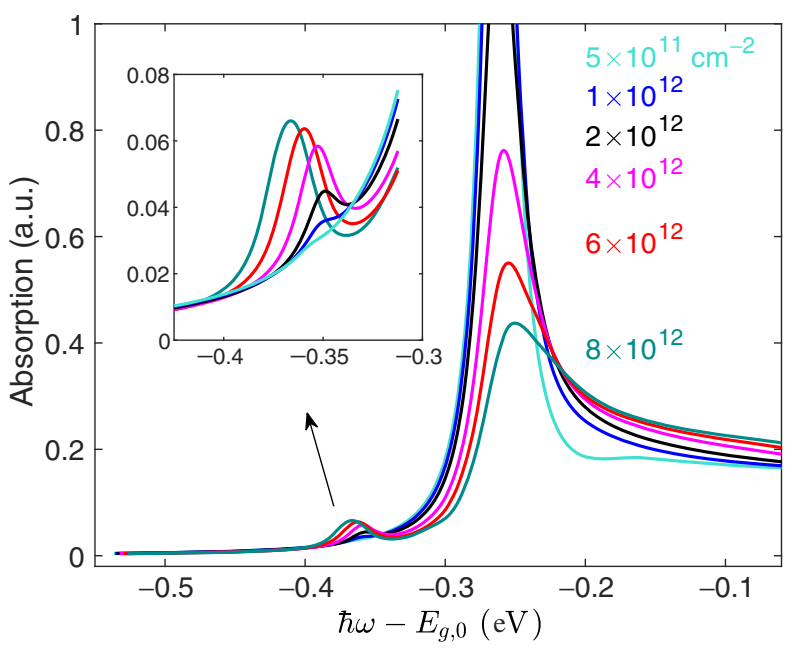

FIG. 8. The absorption spectrum of direct excitons in electrondoped ML-WX $\mathrm{W}_{2}$ after renormalization due to interaction with shortwave plasmons. A dynamical sideband emerges in the lowenergy side of the absorption spectrum, as highlighted in the enlarged box. The sideband initially redshifts and intensifies when the electron density increases, showing saturated behavior at elevated densities.

the optical transition, and in this respect, its amplitude should be compared with that seen in differential reflectivity rather than photoluminescence experiments (we further address this issue later). Comparing the results in Figs. 1(b) and 8, however, we still find two quantitative disagreements. The first one is that the main peak does not completely decay at elevated densities. As explained before, we attribute this disagreement to the fact that broadening effects are introduced only after solving the two-particle Dyson equation (in the Appendix). The second disagreement is that the relative amplitude of the simulated dynamical sideband is about 3 times weaker than the oscillator strength of the optical sideband. We attribute this mismatch to the difficulty to simulate a stronger dynamical sideband through an increase of the integration cutoff in Eq. (17) outside the small window of free-plasmon propagation (e.g., when $\left|K_{0}-q^{\prime}\right| \gtrsim \alpha k_{F}$ ). The reason for this is that the high-order Padé polynomials used to perform analytical continuation, $\Omega \rightarrow E_{\omega}+i \delta$, are notoriously sensitive to minute numerical errors [62].

We now focus on the redshift and initial amplification of the dynamical sideband, which are common to both experiment [as shown in Figs. 1(b)] and our simulations (highlighted in the enlarged box of Fig. 8). The redshift is caused by the electron-electron exchange interaction when the lower spin-split valleys are being filled. This interaction leads to a stronger energy redshift of the populated lower valleys compared with the unpopulated upper ones (inset of Fig. 6), thereby increasing the plasmon energy via $\Delta$ in Eq. (5). The initial amplification of the dynamical sideband is commensurate with the increase in the number of free- 

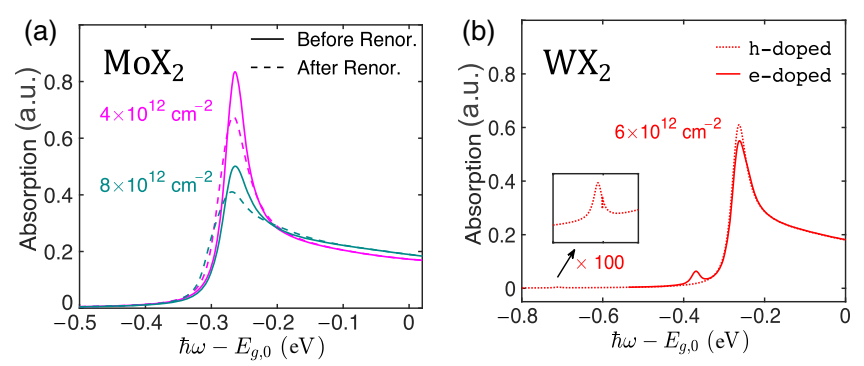

FIG. 9. The renormalized absorption spectrum of direct excitons in ML-MoX ${ }_{2}$ and in the case of hole doping. (a) The case of electron-doped ML-MoX $\mathrm{X}_{2}$ before (solid lines) and after (dashed lines) renormalization. The dynamical sideband is not seen. (b) The case of electron-doped (solid line) and hole-doped (dotted line) ML-WX $\mathrm{W}_{2}$. The dynamical sideband is negligible for hole doping, as highlighted in the magnified box. It emerges about $400 \mathrm{meV}$ below the exciton line because of the large valence-band splitting.

propagating plasmon modes (larger value of $\alpha k_{F} / 3$ ). Saturation at high densities occurs when the energy difference between direct and indirect excitons, $\delta E_{\mathrm{D}-\mathrm{I}}$, deviates from the energy of shortwave plasmons, about $\Delta$. Specifically, indirect excitons experience a blueshift due to electron filling of the lower valleys, while direct ones are not subjected to this effect since they originate from electronic states in the empty upper valleys. Continuing to increase the electron density eventually leads to a slow decay of the dynamical sideband due to increased "off-resonance" conditions between $\delta E_{D-I}$ and $\Delta$ (Appendix A 5).

Our model shows that the dynamical sideband is evident only in electron-doped ML-WX $\mathrm{W}_{2}$, providing strong evidence that it corresponds to the measured optical sideband [9-16]. Figure 9(a) shows that the dynamical sideband is absent in electron-doped ML-MoX ${ }_{2}$, simulated by reversing the order of the conduction-band valleys such that direct excitons have lower energy [Fig. 2(b)]. The dynamical sideband is not seen because of "wrong conditions." Namely, the sideband is positioned at about one plasmon energy below the energy of the indirect exciton, which in the case of ML-MoX ${ }_{2}$, coincides with the direct-exciton spectral line. This behavior makes it hard to resolve the optical signature of exciton-plasmon quasiparticles in ML-MoX ${ }_{2}$. Figure 9(a) indeed shows that the renormalization only affects the spectral region of the exciton peak without the emergence of dynamical sidebands in other spectral regions. In electron-doped $\mathrm{ML}-\mathrm{WX}_{2}$, on the other hand, the dynamical sideband can be distinctly recognized since the indirect exciton has lower energy; thus, overall, the sideband emerges about $2 \Delta$ below the direct-exciton spectral line.

Further evidence that the revealed exciton-plasmon phenomenon corresponds to the optical sideband in electron-doped ML-WX ${ }_{2}$ is provided by simulating the hole- doped case. Figure 9(b) shows the renormalized absorption spectra of hole- and electron-doped ML-WX $\mathrm{W}_{2}$. The dynamical sideband is practically absent in the hole-doped case, which is similar to the experimental findings in Figs. 1(c) and 1(d). We can understand this behavior by noting that for electron doping, the splitting of the conduction-band valleys corresponds to both plasmon energy and the energy difference between direct and indirect excitons (the role of electron-hole exchange is relatively small as we explain in Appendix A 4). For hole doping, on the other hand, the plasmon energy is governed by the large splitting of the valence-band valleys (about $400 \mathrm{meV}$ in ML-WX $\mathrm{X}_{2}$ ), while the energy difference between direct and indirect excitons is still governed by the much smaller splitting in the conduction band. The large mismatch between the two suppresses the emergence of a visible dynamical sideband in hole-doped ML-TMDs.

\section{Stokes vs anti-Stokes optical sidebands}

Figure 8 shows that the exciton-plasmon resonance emerges about $2 \Delta$ below the direct-exciton spectral line in electron-doped ML-WX $\mathbf{W}_{2}$ Figure 9(a) shows that it coincides with the spectral line of the direct exciton in electron-doped ML-MoX ${ }_{2}$. These calculations did not reveal the emergence of an optical sideband above the direct-exciton spectral line because of the small population of shortwave plasmons at the simulated low temperature $(T=10 \mathrm{~K}), \quad g\left(E_{\mathrm{s}}(q)\right) \rightarrow 0 \quad$ when $\quad E_{\mathrm{s}}(q) \sim \Delta \gg k_{B} T$. Specifically, the self-energy of direct excitons includes both plasmon emission and absorption processes, corresponding to "Stokes" and "anti-Stokes" sidebands below and above the energy of the indirect exciton, respectively (see Appendix A 2). The amplitude of the Stokes sideband is commensurate with $-g\left(-E_{\mathrm{s}}(q)\right)=1+g\left(E_{\mathrm{s}}(q)\right)$, while that of the anti-Stokes sideband is commensurate with $g\left(E_{\mathrm{s}}(q)\right)$. The vanishing value of the latter at low temperatures explains why Figs. 8 and 9 do not include signatures of high-energy optical sidebands due to plasmon absorption.

At high temperatures, on the other hand, it may be possible to observe a distinct high-energy optical sideband in the absorption spectrum of electron-doped ML-MoSe 2 . The value of $\Delta$ in this material is non-negligible $[32,56]$, resulting in a well-resolved anti-Stokes sideband whose energy is about $2 \Delta$ above the spectral line of the direct exciton. This high-energy optical sideband is expected to blueshift when the electron density increases because of the increasing value of $\Delta$. Its amplitude, however, should be measurably weaker than that of the Stokes sideband in ML-WX ${ }_{2}$ since the ratio $\left|g\left(E_{\mathrm{s}}(q)\right) / g\left(-E_{\mathrm{s}}(q)\right)\right|$ remains evidently smaller than 1 even close to room temperature (see Appendix A 2). In addition, the observation of a highenergy optical sideband in high-temperature ML-MoSe ${ }_{2}$ may be elusive if it resides close to or within the continuum. In other words, the exciton-plasmon coupling is manifested at elevated electron densities in which the exciton energy 
approaches the continuum because of the strong BGR. The emergence of an exciton-plasmon resonance above the direct-exciton spectral line is hampered by broadening effects, similar to the behavior of neutral excitons when their energy approaches the continuum (Fig. 6). The plasmon emission process, on the other hand, is relatively robust since the increased value of $\Delta$ with electron density causes the low-energy optical sideband to redshift, thereby keeping it protected from merging into the redshifting continuum. Accordingly, broadening effects of the Stokes sideband are mitigated, and ultrafast dissociation of the exciton-plasmon quasiparticle to the continuum of electron-hole pairs is suppressed. Appendix A 2 includes further details on the Stokes and anti-Stokes optical sidebands.

\section{DISCUSSION}

When comparing the results in this work with experimental findings, one should recall that the theory only evaluates the pair function and its renormalization due to coupling with plasmons. Accordingly, the theory does not capture optical transitions that are associated with threebody complexes [21,22,63-70], Fermi polarons [27,28], and exciton optical transitions next to localization centers [71]. Nonetheless, the theory in this work covers two important regimes. The first one is the low-density regime, $n \lesssim 10^{12} \mathrm{~cm}^{-2}$, in which the neutral exciton dominates the absorption spectrum, and its decay is not compensated by an equivalent increase of the trion peak, as shown by the empirical data in Fig. 1(a). The dynamical picture we have developed quantifies the exciton decay due to longwavelength plasmons (Fig. 6), where we find good agreement with reflectivity experiments [15]. The second case is the high-doping regime, $n \gtrsim 5 \times 10^{12} \mathrm{~cm}^{-2}$, in which both reflectivity and PL experiments show that only one peak survives in the spectrum of electron-doped ML-WX 2 $[10,15]$, as shown by Fig. 1(b). Trions cease to exist at very large densities since their effective radius is larger than the average distance between electrons of the Fermi sea. The latter is proportional to $1 / \sqrt{n}$, while the trion extension is of the order of 3-5 nm in ML-TMDs [69,70]. The main focus of this work is on the high-doping regime, and we attribute the observed optical transition to a new type of quasiparticle where the exciton is coupled to a shortwave charge fluctuation. We provided evidence that the behavior of the optical sideband when increasing the electron density matches that of the exciton-plasmon quasiparticle, and we further showed that it emerges in electron-doped ML-WX but not in the hole-doped or ML-MoX ${ }_{2}$ cases (Figs. 8 and 9). Any alternative explanation that aims at deciphering the origin for the optical sideband should be consistent with this observation. Below, we discuss several key aspects that one may find helpful when interpreting experimental findings and when comparing them with our theory.

\section{A. Plasmon-assisted optical transitions}

We have attributed the optical sideband in the reflectance spectra to the creation of an exciton-plasmon quasiparticle. It was found by renormalization of the pair function of direct excitons, through the sum of infinite plasmoninduced virtual transitions to intermediate indirect-exciton states [Fig. 7(b)]. This physical picture should not be confused with that of a plasmon-assisted optical absorption, in which an indirect exciton is created through two virtual transitions into and from a direct exciton. To better understand this difference and its implication in the context of the experimental data (Fig. 1), we assume low temperatures where only emission of a shortwave plasmon is feasible during these processes $\left(\Delta \gg k_{B} T\right)$.

\section{Plasmon-assisted photon absorption (reflectivity spectrum)}

In photon absorption at low temperatures, the plasmonassisted process creates an indirect exciton and a shortwave plasmon in the final state. The overall energy conservation of the absorption process is such that the photon energy is the sum of the indirect exciton and plasmon energies,

$$
\hbar \omega=E_{I}\left(-\mathbf{q}_{f}\right)+E_{\mathrm{s}}\left(\mathbf{q}_{f}\right),
$$

where the crystal momenta of the plasmon and indirect exciton are opposite in order to guarantee momentum conservation (the photon carries negligible momentum). Here, the photon is absorbed into a direct-exciton virtual state, followed by plasmon emission that transfers the exciton to its final state. Crystal momentum must be conserved during virtual transitions to and from intermediate states because of translation symmetry. As a secondorder process, the absorption amplitude is weak unless the energy of the intermediate virtual state resonates with the real energy level of the direct exciton. Recalling the energy diagram in Fig. 2, Eq. (20) implies that this condition is met in electron-doped ML- $\mathrm{WX}_{2}$ but not in $\mathrm{ML}^{-\mathrm{MoX}_{2}}$. In other words, the plasmon-assisted photon absorption is a secondorder resonance process in electron-doped $\mathrm{ML}-\mathrm{WX}_{2}$, meaning that the neutral-exciton spectral line $\left(X_{0}\right)$ has two contributions. The first one is from the first-order absorption process, which creates direct excitons. The second contribution is from the second-order resonance process, which creates indirect excitons via emission of shortwave plasmons.

Therefore, an important resulting question is how one can resolve the relative contribution of the plasmon-assisted optical transitions to the oscillator strength of $X_{0}$ in electron-doped $\mathrm{ML}-\mathrm{WX}_{2}$. Clearly, the contribution has negligible weight at vanishing electron densities because of the minute range of free-propagating plasmon modes. When the charge density increases, however, we can identify this contribution by looking for differences between the reflectance data in the electron-doped and 
hole-doped regimes. Indeed, Figs. 1(a) and 1(c) reveal a clear difference in the measured behavior of $X_{0}$, whose blueshift and broadening are evidently stronger in the electron-doped case. As we suggest below, the plasmonassisted optical transitions can give rise to this behavior.

Without the contribution of shortwave plasmons, one would expect the blueshift of $X_{0}$ to be stronger in the holedoped case. Specifically, the blueshift of neutral excitons in the absorption spectrum of semiconductors is induced by filling one or both of the bands that are involved in the optical transition [54]. This condition is not readily met in electron-doped ML-WX $\mathrm{X}_{2}$ since only the bottom conduction valleys are populated, whereas the optical transition involves the top valleys. Both experiment and theory show that electron population in the top valleys of ML-WX begins at densities that are comparable to $10^{13} \mathrm{~cm}^{-2}$ $[15,32]$. The situation is different for the hole-doped case at which hole filling in the top of the valence band is measurable at smaller densities. Thus, while such behavior should lead to weaker or at most comparable blueshift of the direct exciton in the electron-doped case, the experiment shows the opposite. Furthermore, the simulated decay of the pair function in Fig. 6 better resembles the observed decay of $X_{0}$ in the hole-doped case. This fact suggests a missing component in the theory such that dynamical screening and BGR are not enough to fully explain the behavior of $X_{0}$ in electron-doped ML-WX .

Plasmon-assisted optical transitions can complete the puzzle and explain the stronger blueshift and broadening of $X_{0}$ in electron-doped ML-WX $\mathrm{W}_{2}$. There are two complementing facts that support this claim. First, plasmonassisted optical absorption stems from a resonance process in electron-doped ML-WX $\mathrm{W}_{2}$, suggesting a non-negligible effect. Second, Eq. (20) can explain the enhanced blueshift in the experiment since the plasmon energy increases at elevated densities (through the increased value of $\Delta$ ). Finally, the enhanced broadening of $X_{0}$ in ML-WX $X_{2}$ when the electron density increases can be explained by the closer proximity of the photon energy to the redshifting continuum (or possibly merging with the continuum). We will provide a complete description of these phenomena alongside second-order perturbation theory to describe the plasmon-assisted optical transitions in a future work.

All in all, the optical sideband in the reflectance experiment of electron-doped ML-WX $\mathrm{W}_{2}\left(X-^{\prime}\right)$ is attributed to the exciton-plasmon quasiparticle, originating from renormalization of the self-energy of the direct exciton. Its energy is one (two) plasmon(s) below that of the indirect (direct) exciton. Photon absorption due to plasmon-assisted optical transitions, on the other hand, is a second-order process that converts photons into indirect excitons through plasmon emission at low temperatures. It becomes important in electron-doped ML-WX ${ }_{2}$, in which the intermediate virtual exciton state resonates with the real energy level of the direct exciton. The signature of this process in the reflectance experiment of electron-doped ML- $\mathrm{WX}_{2}$ is limited to the spectral region of the direct exciton $\left(X_{0}\right)$. Unlike phononassisted optical transitions [32,72-74], the amplitude of the plasmon-assisted ones increases with the electron density because of the increased range of free-propagating plasmon modes.

\section{Plasmon-assisted photon emission (PL spectrum)}

In photon emission at low temperatures, the plasmonassisted process creates a shortwave plasmon and a photon in the final state through annihilation (radiative recombination) of an indirect exciton. The overall energy conservation of the emission process is such that

$$
\hbar \omega=E_{I}\left(-\mathbf{q}_{f}\right)-E_{\mathrm{s}}\left(\mathbf{q}_{f}\right) .
$$

Here, the indirect exciton in the initial state is transitioned into a direct-exciton virtual state by plasmon emission, followed by the photon emission. Recalling the energy diagram in Fig. 2, Eq. (21) implies that the resonance condition is now met in electron-doped $\mathrm{ML}_{-} \mathrm{MoX}_{2}$, in which the energy of the intermediate virtual state resonates with the real energy level of the direct exciton. However, this second-order resonance process has no real value at low-temperature PL since the population of indirect excitons in ML-MoX $\mathrm{X}_{2}$ is negligible at low temperatures: Most excitons in these compounds remain direct after photoexcitation and energy relaxation [Fig. 2(b)]. Therefore, we do not expect plasmon-assisted optical transitions to have a measurable signature in the PL spectrum of $\mathrm{ML}-\mathrm{MoX}_{2}$ at low temperatures.

The case of electron-doped ML-WX $\mathrm{W}_{2}$ is unique if we consider the renormalization of direct excitons that led to the exciton-plasmon quasiparticle. In the reflectance spectrum, we have concluded that the contributions from the creation of indirect excitons through the plasmon-assisted photon absorption and the creation of exciton-plasmon quasiparticles are spectrally resolved: The latter corresponds to the optical sideband $\left(X-^{\prime}\right)$, and the former can only affect the spectral region of the bare neutral exciton $\left(X_{0}\right)$. In the PL spectrum, on the other hand, Eq. (21) suggests that the photon energy should coincide with that of the optical sideband. In other words, both the annihilation of the exciton-plasmon quasiparticle and the annihilation of indirect excitons through plasmon-assisted photon emission contribute to $X-^{\prime}$ in the PL spectrum. The latter contribution may not be negligible because of a large population of indirect excitons after energy thermalization in $\mathrm{ML}^{-W X} \mathrm{~W}_{2}$ [Fig. 2(a)]. In addition, while the energy of the intermediate virtual state does not resonate with the real energy level of the bare direct exciton, it matches the low energy of the dressed one (i.e., the exciton-plasmon quasiparticle). Therefore, the recombination of indirect excitons due to plasmon-assisted photon emission may have a measurable signature on the spectral weight of $X-^{\prime}$, explaining in part why its magnitude is much stronger in $\mathrm{PL}$ 
compared with reflectance spectra (see related discussion in Sec. I) $[10,15]$. We note that modeling of the densitydependent PL spectrum is beyond the scope of this work, partly because of the lack of knowledge of the nonradiative recombination processes. For example, charged impurities that function as nonradiative recombination centers are screened at elevated densities, resulting in improved quantum efficiency for radiative recombination. Conversely, nonradiative Auger processes are amplified at elevated densities [75-77], thereby reducing the quantum efficiency.

\section{High temperatures}

So far, we have discussed plasmon-assisted optical transitions at low temperatures, in which only emission of shortwave plasmons is possible during the photon absorption or emission. At high temperatures, on the other hand, we should also consider the absorption of shortwave plasmons during these optical processes. Repeating the previous analyses, one can see that the conditions for second-order resonance processes are switched between ML-MoX ${ }_{2}$ and ML-WX $\mathrm{X}_{2}$. Detecting the signatures of these processes in the high-temperature reflectance or PL spectra may be elusive for the same reasons we have mentioned regarding the anti-Stokes optical sideband in Sec. III C.

\section{Other optical spectroscopy tools to probe the plasmon-exciton coupling}

In addition to differential reflectivity and photoluminescence experiments, it may be possible to probe the coupling between excitons and shortwave plasmons in electrondoped samples using Raman-type experiments or nonlinear optical spectroscopy. For example, the energy of scattered photons in the Stokes sideband of a Raman experiment is expected to be $2 \Delta$ below that of the incident photon. It involves a virtual process through which two counterpropagating shortwave plasmons are emitted, so the sum of their momenta becomes negligible and matches the minute momentum of photons. In the first step of this process, the incident photon induces a virtual transition to a direct exciton intermediate level, which in the second step is scattered to the indirect exciton intermediate level because of plasmon emission. In the next step, the indirect exciton is scattered back to the direct exciton intermediate level by emission of a counterpropagating plasmon. Finally, the system emits a photon at the Stokes energy (the energy of the incident photon minus the energy of two plasmons). The overall process is expected to be weak since two plasmon-mediated virtual transitions are involved. The amplitude of the scattered light can increase by using near-resonance conditions. The amplitude of the scattered light and its energy can be controlled by employing a gate voltage that tunes the value of $\Delta$ through electron filling of the lower spin-split valleys. In other words, the gate voltage provides an additional control parameter in the experiment. A similar approach can be used in nonlinear wave-mixing spectroscopy: The generated wave is amplified when the detuning between the input beams approaches $2 \Delta$.

\section{B. Three-body complexes versus exciton-plasmon quasiparticles}

It is beyond the scope of this work to provide a complete three-body dynamical function since it is impossible to numerically solve its corresponding Dyson equation without simplifying approximations. The dynamical model we have developed describes the behavior of the pair function and its renormalization due to coupling with shortwave plasmons. The optical sideband, which we have associated with the exciton-plasmon quasiparticle, has nearly opposite behavior from that of charged excitons (see discussion of Fig. 1 in Sec. I). Accordingly, the identification of charged excitons complexes and exciton-plasmon quasiparticles can be readily achieved. Studying how the dynamically screened potential influences the oscillator strength and broadening of charged excitons is a work in progress. Here, we mention some of the known theoretical approaches to study their behavior. The most computationally convenient way to deal with trions is to dispense with the dynamical terms altogether [20,63-68], but then one can only conclude about their binding energy. Such static models cannot describe the density-dependent rise, decay, energy shift, and broadening of trions in the spectrum. Alternatively, one can neglect dynamical terms in the exciton-electron interaction [27] or a priori assume the dynamical form of the Green's function without solving the corresponding dynamical Dyson equation $[21,28]$. In the latter case, one can describe the behavior of charged excitons by invoking an exciton-electron scattering function, where dynamical terms in the scattering potential are neglected.

One open question in the context of our work deals with possible coupling between trions and shortwave plasmons. Figures 1(a) and 1(b) do not show optical sidebands whose energy is $\Delta$ or $2 \Delta$ below that of the charged exciton spectral line; the value of $\Delta$ is about $26 \mathrm{meV}$ at low densities [61], and it increases at elevated ones [35]. The absence or weakness of trion-plasmon coupling is attributed to the relatively small oscillator strength of the charged exciton compared with that of the neutral exciton (Fig. 1). The oscillator strength is inversely proportional to the square of the complex radius, where the radius is about 3 times larger for trions $[69,78]$. This fact is also reflected in the much smaller binding energy of the charged exciton. Here, we reemphasize that the decay of the neutral-exciton spectral line with the increase in electron density is mostly caused by broadening effects when the exciton energy approaches the redshifting continuum (see discussion of Fig. 6). The quasiparticle made by a neutral exciton coupled to a shortwave plasmon is resilient to this broadening since its energy is kept away from the redshifting continuum due to the increasing value of $2 \Delta$ when the density is ramped up. For that reason, the oscillator strength of the optical 
sideband neither decays nor broadens at elevated densities [Fig. 1(b) and Fig. 8]. Quantitatively, the oscillator strength amplitude is manifested in a relatively large value of the pair function at even Matsubara energies, compared with the respective dynamical Green's function that would describe charged excitons. Finally, we mention that the optical sideband is weak but noticeable even when $E_{F}$ is significantly smaller than the binding energy of the charged exciton [enlarged boxes of Figs. 1(a) and 8]. In this regime, the negatively charged exciton manages to stay away from electrons and plasma excitations of the Fermi sea, as evident from the absence of broadening and blueshift of $X_{-}^{A}$ and $X_{-}^{B}$ in Fig. 1(a). Neutral electron-hole pairs, on the other hand, are not repelled by the surrounding Fermi sea and therefore can be coupled to shortwave plasmons at all finite densities.

\section{Strong photoexcitation conditions}

Recently, You et al. observed the emergence of a strong spectral line in the PL of ML-WSe 2 when subjected to strong excitation [79]. This peak showed quadratic dependence on the intensity of the pump signal and did not shift appreciably when increasing the pumping intensity. This behavior suggests that the emerged spectral line is due to excitonexciton interactions [79]. A similar behavior was also observed in the PL of ML-WS 2 [11,12]. To date, however, this behavior was not reported in the PL of ML-MoX ${ }_{2}$.

We argue that these observations can be settled by considering the shortwave Coulomb excitations. Specifically, the strong pump intensity generates a relatively large density of direct excitons, the majority of which relax in energy and become indirect in ML-WX $\mathrm{W}_{2}$ [Fig. 2(a)] [32,33]. The band structure of indirect excitons is similar to the one shown in Fig. 4(a) [7], where their electron component generates the same type of shortwave Coulomb excitations. A direct exciton can then interact with these excitations in the same way as before, leading to renormalization of its self-energy. The observed biexciton spectral line emerges through this renormalization. The main difference from the optical sideband, which emerges under conditions of weak photoexcitation in electron-doped ML-WX $\mathrm{W}_{2}$, is that now the pumping is responsible not only for the exciton population but also for the generation of shortwave Coulomb excitations through the electron components of indirect excitons. These two responsibilities explain the quadratic dependence of the observed optical transition on the pumping intensity. In this view, the measured phenomena can still be identified as an excitonexciton interaction but with the notion that the coupling is between direct and indirect excitons due to shortwave charge fluctuations.

The importance of our theory is therefore in suggesting a microscopic origin for the exciton-exciton optical transition in the PL of strongly photoexcited ML-WX $\mathrm{W}_{2}$. We claim that it originates from the intervalley Coulomb excitations and not from other sources such as van der Waals-type attraction between two neutral excitons. If the latter was the microscopic origin for the observed biexciton spectral line, then it should be insensitive to whether the two excitons are direct, indirect, or a mixture of the two. As a result, one should be able to observe the biexciton spectral line in the PL of both ML-MoX ${ }_{2}$ and $\mathrm{ML}-\mathrm{WX}_{2}$ under comparable pumping intensities. The fact that this scenario is not observed in experiments precludes such microscopic origins. Furthermore, previous theoretical calculations have shown that the binding energy of the biexciton is about $20 \mathrm{meV}$ either in ML-MoX ${ }_{2}$ or ML-WX $\mathrm{W}_{2}$, by using a variety of four-body computational models with static 2D Coulomb (Keldysh) potentials [63,65,69,78,80-82]. PL experimental reports, on the other hand, are available only for $\mathrm{ML}^{-\mathrm{WX}_{2}}$ in which the biexciton emerges about 50-60 meV below the neutral-exciton spectral line $[11,12,79]$. Our claim regarding the coupling between direct and indirect excitons through shortwave charge fluctuations is indeed self-consistent with the fact that the biexciton spectral line has not been reported to date in PL experiments of ML-MoX ${ }_{2}$. The low-temperature population of indirect excitons in these compounds is negligible because of their higher energy, as shown in Fig. 2(b). Moreover, the coupling between indirect and direct excitons has the same wrong conditions that were discussed in the context of Fig. 9(a). Namely, the renormalization of direct excitons does not lead to a distinct spectral line, which can be resolved from that of the neutral exciton.

In addition, our explanation is consistent with the fact that the indirect-direct biexciton spectral line in ML-WX emerges at the same spectral position of the optical side-

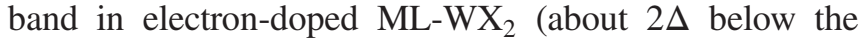
direct-exciton spectral line, which is of the order of about $50-60 \mathrm{meV}$ in these materials) $[10,11,15,16]$. One difference between the two cases, however, is that the energy shift of the direct-indirect biexciton is weak when the photoexcitation intensity increases [79], compared with the redshift of the optical sideband when increasing the electron density [10]. The reason for this is that, unlike gateinduced free electrons, bound and neutral excitons do not lead to an appreciable change of $\Delta$. The value of $\Delta$ can change because of the long-wavelength exchange interaction, which is effective in the case of free electrons [35]. As a result, the indirect-direct biexciton shows a relatively weak energy shift when increasing the photoexcitation intensity (as long as excitons remain bound).

\section{CONCLUSION AND PERSPECTIVES}

The identified pairing of shortwave plasmons and excitons explains the puzzling observations of the emerging low-energy optical sideband in the spectra of electrondoped $\mathrm{WSe}_{2}$ and $\mathrm{WS}_{2}$, while being conspicuously absent with hole doping, $\mathrm{MoSe}_{2}$ and $\mathrm{MoS}_{2}$ [9-16]. Elucidating the exciton-plasmon mechanism and the importance of dynamical screening paves the way to harness unexplored 
manifestations of spin-valley coupling and the realization of other dynamical sidebands, such as those arising because of the exciton-phonon coupling or the coupling between zone-edge phonons and intervalley plasmons.

The findings of this work reveal the unique nature of shortwave (intervalley) plasmons in monolayer transitionmetal dichalcogenides. Recalling that freely propagating shortwave plasmons are independent d.o.f. in the electronic system, these quasiparticles can be directly detected in electron-rich monolayers (i.e., not through their coupling to excitons). Using $\mathrm{THz}$ spectroscopy, for example, one should expect to see a resonance in the absorption spectrum when the photon energy is about twice the spin splitting in the conduction band $(\lesssim 0.1 \mathrm{eV})$. Such far-infrared photons can couple to two counterpropagating shortwave plasmons, thereby conserving both energy and momentum. As before, the plasmon energy and amplitude can be tuned by a gate voltage. In addition, we expect dynamical screening to be important in the emerging area of magnetic proximity effects in monolayer transition-metal dichalcogenides [83]. It has been experimentally shown that magnetic substrates can strongly alter the optical response of excitons, induced by time-reversal symmetry breaking between the $K$ and $-K$ valleys [84-86]. Equivalently, magnetic proximity effects or strong magnetic fields should lead to symmetry breaking between counterpropagating shortwave plasmons.

Beyond monolayer transition-metal dichalcogenides, virtual exchange of shortwave plasmons may give rise to pairing mechanisms in superconductors whose Fermi surfaces comprise distinct pockets in the Brillouin zone. Similar to our formalism, plasmon-mediated Cooper pairing can be modeled when a dynamical screening description is employed [87-90], and it was argued to facilitate superconductivity in cuprate metal oxides [91] and other transition-metal systems through plasmon-phonon hybridization [92,93], as well as in semiconductors and electronhole liquids [94-97].

\section{ACKNOWLEDGMENTS}

The authors are indebted to Zefang Wang, Kin Fai Mak, and Jie Shan for providing the reflectivity measurements in Fig. 1 and for many useful discussions. We also thank Xiaodong $\mathrm{Xu}$ for discussing and sharing valuable experimental results prior to their publication. This work is mainly supported by the Department of Energy, Basic Energy Sciences (Grant No. DE-SC0014349). The computational work at Rochester was also supported by the National Science Foundation (Grant No. DMR-1503601) and the Defense Threat Reduction Agency (Grant No. HDTRA1-131-0013). The work at Buffalo was supported by the Department of Energy, Basic Energy Sciences (Grant No. DE-SC0004890). The work at Regensburg and Würzburg was supported by the DFG (Grants No. SCHA 1899/2-1 and No. SFB 1170 "ToCoTronics"), and by the ENB Graduate School on Topological Insulators.

\section{APPENDIX: COMPUTATIONAL DETAILS}

Equation (9) is solved with matrix inversion. Each row (or column) in the matrix is indexed via $\left\{k_{i}, z_{n}\right\}$. The angular dependence of $\mathbf{k}_{i}-\mathbf{k}_{2}$ in $V_{s}\left(\mathbf{k}_{i}-\mathbf{k}_{2}, z-z^{\prime}\right)$ is averaged out, and then all the $2 \mathrm{D}$ wave vectors in Eq. (9) are treated as scalars. We use a uniform grid with a spacing of $\Delta k=k_{\max } / N_{k}$, where $k_{\max }$ is chosen such that $\varepsilon_{k_{\max }}=1 \mathrm{eV}$. For the fermion Matsubara energies, $z_{n}=$ $i \pi\left(2 n_{z}+1\right) k_{B} T, n_{z}$ runs between $-N_{z}$ to $+N_{z}$. The boson Matsubara energy $\Omega$ in Eq. (9) is treated as a parameter in the matrix. In other words, we solve this equation for each $\Omega_{n}=i 2 \pi n_{\Omega} k_{B} T$ with $n_{\Omega} \in\left[-N_{\Omega}, N_{\Omega}\right]$. For numerical calculations, we have used $N_{k}=50, \quad N_{z}=160$, $N_{\Omega}=90$, and the code was written in FORTRAN and parallelized with OpenMP. For every density, the calculation of $G_{p}(q=0, k, z, \Omega)$ or $G_{p}\left(q=K_{0}, k, z, \Omega\right)$ takes about 7 hours when using 44 CPUs $(2 \mathrm{GHz})$ with $1 \mathrm{~TB}$ shared DRAM.

After the calculation of the pair Green's function, we use Eq. (6) to calculate the absorption spectrum. Specifically, we first calculate the function

$$
A(\Omega)=\beta^{-1} \sum_{z} \operatorname{Im}\left\{\int d k k G_{p}(q=0, k, z, \Omega)\right\},
$$

and then use Padé polynomials to extract the analytical form of $A\left(\Omega \rightarrow E_{\omega}+i \delta\right)$ in the upper complex plane from knowledge of its values at discrete even Matsubara energies [52]. The broadening term is taken as [54]

$$
\delta \equiv \delta\left(E_{\omega}\right)=\delta_{1}+\frac{\delta_{2}}{1+\exp \left[\left(E_{c}-E_{\omega}\right) / \delta_{3}\right]},
$$

where $\delta_{j}(j=\{1,2,3\})$ are three parameters with the following meaning: $\delta_{1}$ reflects the inhomogeneous broadening due to disorder, adatoms, and charge puddles; $\delta_{2}$ and $\delta_{3}$ reflect the enhanced homogeneous broadening when the transition energy belongs to the continuum $\left(E_{\omega}>E_{c}\right)$. Far below the continuum, we get $\delta\left(E_{\omega}\right) \rightarrow \delta_{1}$. Far above the continuum edge, we get $\delta\left(E_{\omega}\right) \rightarrow \delta_{1}+\delta_{2}$. See Chapter 13 in Ref. [54] for more details. In this work, we have used $\delta_{1}=10 \mathrm{meV}, \delta_{2}=70 \mathrm{meV}$, and $\delta_{3}=20 \mathrm{meV}$.

The results shown in Figs. 8 and 9 were obtained using the renormalization in Eq. (16). The self-energy term, whose expression is provided in Eq. (17), is calculated by summing over even Matsubara energies and by integrating over $\mathbf{q}^{\prime}$. The integration interval for the latter is $\left|\mathbf{q}^{\prime}-\mathbf{K}_{\mathbf{0}}\right| \lesssim \gamma(\alpha / 3) k_{F}$, where $\gamma=2$. In other words, we have assumed that plasmons are not immediately damped outside the region of free-plasmon propagation.

\section{Effective mass model}

We emphasize that the goal of this work is not to reproduce the exact binding energy of excitons in 
ML-TMDs. Rather, we are interested in the effects of dynamical screening and coupling between excitons and shortwave plasmons. We use the effective mass approximation since it allows us to simplify the calculation while keeping the main dynamical effects intact. In the calculation of the pair functions of direct and indirect excitons, we have assumed similar effective masses for electrons in the lower and upper valleys of the conduction band. We ignore the (small) change in the values of the effective masses at the edges of the lower and upper valleys since the binding energy of excitons in ML-TMDs is much larger than the conduction-valley splitting energy. In other words, the matrix we use to solve the pair function includes electronic states whose energies are hundreds of meV above the edge of the band. For such states, the exact value of the effective mass in the bottom of the band is meaningless. We therefore choose the same value for both lower and upper valleys, which represents the effective mass of the "extended" conduction band.

\section{Emission and absorption of plasmons}

The self-energy of the exciton due to the interaction with shortwave plasmons, given in Eq. (17), includes both emission and absorption terms. Formally, these terms are derived from the identity [36,51]

$$
\begin{aligned}
& \beta^{-1} \sum_{\Omega^{\prime}} D\left(\Omega-\Omega^{\prime}, \mathbf{q}^{\prime}\right) G_{p}\left(\mathbf{q}^{\prime}, \mathbf{k}, \Omega^{\prime}\right) \\
& =\frac{1}{2 \pi i} \oint_{C} d \Omega^{\prime} \frac{1}{\exp \left(\beta \Omega^{\prime}\right)-1} D\left(\Omega-\Omega^{\prime}, \mathbf{q}^{\prime}\right) G_{p}\left(\mathbf{q}^{\prime}, \mathbf{k}, \Omega^{\prime}\right),
\end{aligned}
$$

where the sum runs over even Matsubara energies, and the integration contour $C$ in the upper plane encircles the poles in the positive sense. Using Cauchy's residue theorem, the Bose-Einstein distribution factors for absorption and emission, $g\left( \pm E_{\mathrm{s}}(q)\right)$, result from the poles in the plasmon propagator, $\Omega^{\prime}=\Omega \pm E_{\mathrm{s}}(q)$ [see Eq. (19)], where $\exp (\beta \Omega)=\exp (2 \pi i n)=1$. Furthermore, by approximating the pair function as a simple pole expression, $G_{p} \propto\left(\Omega^{\prime}-E_{0}\right)^{-1}$, where $E_{0}$ is the exciton energy, we can analytically derive the Stokes and anti-Stokes sidebands. The former stems from the emission term $\propto g\left(-E_{\mathrm{s}}(q)\right) /\left[\Omega+E_{\mathrm{s}}(q)-E_{0}\right], \quad$ where $\quad \Omega \rightarrow \hbar \omega+i \delta$ and $g\left(-E_{\mathrm{s}}(q)\right) \rightarrow 1$ at low temperatures. Similarly, the absorption term stems from $\propto g\left(E_{\mathrm{s}}(q)\right) /\left[\Omega-E_{\mathrm{s}}(q)-E_{0}\right]$.

Section III C describes some of the difficulties that may arise when one tries to observe the anti-Stokes sideband in the absorption spectrum of high-temperature ML-MoSe 2 . Other difficulties that impede its detection may be caused by plasmon damping due to coupling with phonons and by the relatively small population of shortwave plasmons due to the increased value of $E_{\mathrm{s}}(q) \sim \Delta$ at elevated electron densities. This behavior can be understood by inspecting the Bose-Einstein distribution factors that are associated with the distinct anti-Stokes and Stokes sidebands, $\left|g\left(\Delta_{M o}\right) / g\left(-\Delta_{W}\right)\right|$, which emerge in ML-MoSe 2 and $\mathrm{ML}-\mathrm{WX}_{2}$, respectively. For example, assuming that these optical sidebands persist at $200 \mathrm{~K}$ and assigning $\Delta \sim$ $40 \mathrm{meV}$ for both materials at some comparable elevated densities [35], the amplitude of the Stokes sideband becomes about 20 times stronger than that of the antiStokes sideband.

\section{Type B excitons}

Type B excitons correspond to optical transitions from the lower spin-split valence band. We expect the coupling between type B excitons in ML-TMDs and shortwave plasmons to be weak because of the ultrashort lifetime of type B excitons: They experience ultrafast intervalley spinconserving energy relaxation of holes to the lower spinsplit valleys. Furthermore, at elevated charge densities in which the plasma excitations become relevant, the bandgap renormalization is strong enough so that type B optical transitions are within the continuum. In other words, when the densities of resident holes or electrons are larger than about $10^{12} \mathrm{~cm}^{-2}$, type $\mathrm{B}$ excitons can experience ultrafast dissociation to free electrons and holes. The coupling to shortwave plasmons is less effective in this case.

\section{Electron-hole exchange}

The relatively large binding energy of excitons in MLTMDs can lead to evident energy splitting between bright and dark excitons [98-100]. The theory presented in our work, however, involves only bright excitons (singlet spin configuration of the electron and hole). Therefore, both direct and indirect bright excitons should experience similar effects due to the electron-hole exchange interaction. As a result, the energy splitting between direct and indirect excitons remains equivalent to the spin splitting of the conduction band. Furthermore, the emergence of the dynamical sideband is relevant at elevated charge densities in which the wave functions of excitons are blown up because of screening (smaller binding energy). The electron-hole exchange is a weaker effect at such conditions.

\section{More simulation results}

It is important to realize that the effects of dynamical screening and interaction with plasmons are largely independent of the exact parameters that we choose for the effective mass and dielectric constant. For example, in the main text, we have assumed that the monolayer is embedded in hexagonal boron nitride, for which there is discrepancy in the reported values of the dielectric constant. Recent experiments use values of $\epsilon=2.7[15,16]$, and we have used this parameter in Figs. 6, 8, and 9. However, previous studies of hexagonal boron nitride reported values that are nearly twice as large, $\epsilon=5.06$ [101]. We have used 


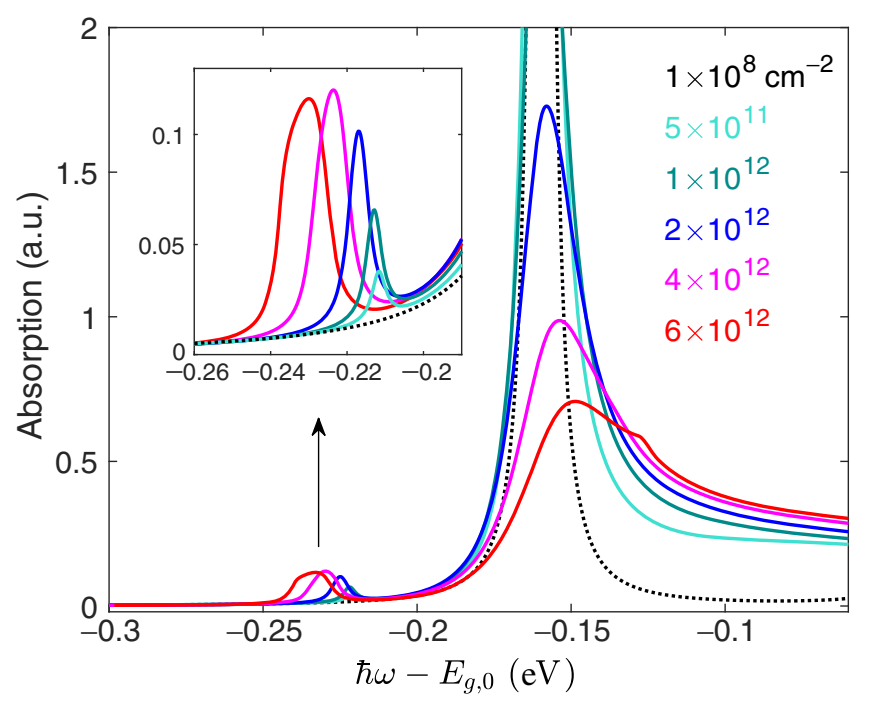

FIG. 10. Renormalized absorption spectrum in electron-doped $\mathrm{ML}-\mathrm{WX}_{2}$ embedded in layers with a dielectric constant, $\epsilon_{b}=\epsilon_{t}=5.06$. This figure shows the same qualitative behavior as in Fig. 8 in which we have used $\epsilon_{b}=\epsilon_{t}=2.7$.

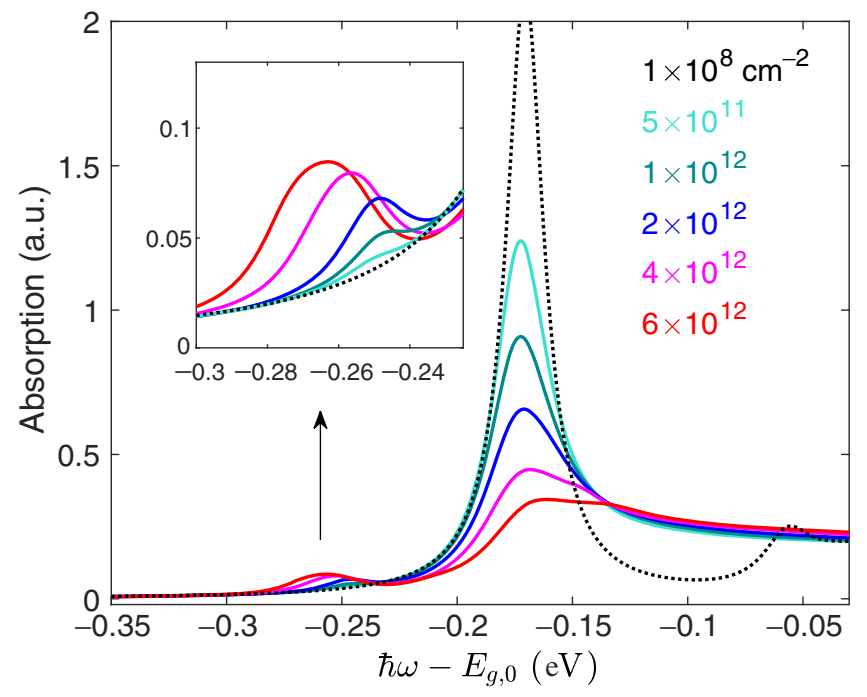

FIG. 11. Renormalized absorption spectrum in electron-doped ML-WX ${ }_{2}$ embedded in layers with a dielectric constant, $\epsilon_{b}=\epsilon_{t}=3.4$. This figure shows the same qualitative behavior as in Figs. 8 and 10. Here, the effective thickness and dielectric constant of the monolayer are $d=0.6 \AA$ and $\epsilon_{\mathrm{ML}}=16.3$, respectively.

this value in Fig. 10, illustrating that such changes do not affect the findings of this work. The only change, as seen from the values of the $x$ axis, is a smaller binding energy due to the larger dielectric screening. Yet, the roles of dynamic screening and the emergence of the dynamical sidebands are the same. Here, we have also used a slightly larger cutoff, $\left|\mathbf{q}^{\prime}-\mathbf{K}_{\mathbf{0}}\right| \lesssim \gamma(\alpha / 3) k_{F}$, where $\gamma=3$ instead of $\gamma=2$, which we used in Figs. 8 and 9. We use this value to enable stronger pairing between excitons and shortwave plasmons, as can be seen from the shoulder and enhanced blueshift of the main peak at elevated densities. At the same time, the redshift of the dynamical sideband is also somewhat enhanced, and it shows slight decay at elevated densities. Finally, Fig. 11 shows the absorption spectrum when using $\gamma=2$ and the effective isotropic model for the values of the thickness and dielectric constant of the ML [102]: $d=0.6 \AA$ and $\epsilon_{\mathrm{ML}}=16.3$. The behavior of the optical sideband is essentially the same, providing further evidence that the exciton-plasmon is a dynamical effect with no qualitative dependence on the exact parameter values that one uses in the static dielectric function.

[1] K. F. Mak, C. Lee, J. Hone, J. Shan, and T. F. Heinz, Atomically Thin $\mathrm{MoS}_{2}$ : A New Direct-Gap Semiconductor, Phys. Rev. Lett. 105, 136805 (2010).

[2] A. Splendiani, L. Sun, Y. Zhang, T. Li, J. Kim, C.-Y. Chim, G. Galli, and F. Wang, Emerging Photoluminescence in Monolayer $\mathrm{MoS}_{2}$, Nano Lett. 10, 1271 (2010).

[3] Q. H. Wang, K. Kalantar-Zadeh, A. Kis, J. N. Coleman, and M.S. Strano, Electronics and Optoelectronics of Two-Dimensional Transition Metal Dichalcogenides, Nat. Nanotechnol. 7, 699 (2012).

[4] A. K. Geim and I. V. Grigorieva, Van der Waals Heterostructures, Nature (London) 499, 419 (2013).

[5] K. F. Mak and J. Shan, Photonics and Optoelectronics of 2D Semiconductor Transition Metal Dichalcogenides, Nat. Photonics 10, 216 (2016).

[6] D. Xiao, G.-B. Liu, W. Feng, X. Xu, and W. Yao, Coupled Spin and Valley Physics in Monolayers of $\mathrm{MoS}_{2}$ and Other Group-VI Dichalcogenides, Phys. Rev. Lett. 108, 196802 (2012).

[7] Y. Song and H. Dery, Transport Theory of Monolayer Transition-Metal Dichalcogenides through Symmetry, Phys. Rev. Lett. 111, 026601 (2013).

[8] K. F. Mak, K. L. McGill, J. Park, and P. L. McEuen, The Valley Hall Effect in $\mathrm{MoS}_{2}$ Transistors, Science 344, 1489 (2014).

[9] X. Xu, W. Yao, D. Xiao, and T.F. Heinz, Spin and Pseudospins in Layered Transition Metal Dichalcogenides, Nat. Phys. 10, 343 (2014).

[10] A. M. Jones, H. Yu, N. J. Ghimire, S. Wu, G. Aivazian, J. S. Ross, B. Zhao, J. Yan, D. G. Mandrus, D. Xiao, W. Yao, and X. Xu, Optical Generation of Excitonic Valley Coherence in Monolayer WSe ${ }_{2}$, Nat. Nanotechnol. 8, 634 (2013).

[11] J. Shang, X. Shen, C. Cong, N. Peimyoo, B. Cao, M. Eginligil, and T. Yu, Observation of Excitonic Fine Structure in a 2D Transition-Metal Dichalcogenide Semiconductor, ACS Nano 9, 647 (2015).

[12] G. Plechinger, P. Nagler, J. Kraus, N. Paradiso, C. Strunk, C. Schüller, and T. Korn, Identification of Excitons, Trions and Biexcitons in Single-Layer $\mathrm{WS}_{2}$, Phys. Status Solidi RRL 9, 457 (2015).

[13] G. Plechinger, P. Nagler, A. Arora, A. Granados del Águila, M. V. Ballottin, T. Frank, P. Steinleitner, M. 
Gmitra, J. Fabian, P. C. M. Christianen, R. Bratschitsch, C. Schüller, and T. Korn, Excitonic Valley Effects in Monolayer $\mathrm{WS}_{2}$ under High Magnetic Fields, Nano Lett. 16, 7899 (2016).

[14] G. Plechinger, P. Nagler, A. Arora, R. Schmidt, A. Chernikov, A. Granados del Águila, P. C. M. Christianen, R. Bratschitsch, C. Schüller, and Tobias Korn, Trion Fine Structure and Coupled Spin-Valley Dynamics in Monolayer Tungsten Disulfide, Nat. Commun. 7, 12715 (2016).

[15] Z. Wang, L. Zhao, K. F. Mak, and J. Shan, Probing the Spin-Polarized Electronic Band Structure in Monolayer Transition Metal Dichalcogenides by Optical Spectroscopy, Nano Lett. 17, 740 (2017).

[16] Z. Wang. K. F. Mak and J. Shan, Valley- and SpinPolarized Landau Levels in Monolayer $\mathrm{WSe}_{2}$, Nat. Nanotechnol. 12, 144 (2017).

[17] M. Koperski, M. R. Molas, A. Arora, K. Nogajewski, A. O. Slobodeniuk, C. Faugeras, and M. Potemski, Optical Properties of Atomically Thin Transition Metal Dichalcogenides: Observations and Puzzles, Nanophotonics 6, 1289 (2017).

[18] A. L. Efros, Metal-Non-Metal Transition in Heterostructures with Thick Spacer Layers, Solid State Commun. 70, 253 (1989).

[19] A. M. Jones, H. Yu, J. Schaibley, J. Yan, D. G. Mandrus, T. Taniguchi, K. Watanabe, H. Dery, W. Yao, and X. Xu, Excitonic Luminescence Upconversion in a TwoDimensional Semiconductor, Nat. Phys. 12, 323 (2016).

[20] E. Courtade, M. Semina, M. Manca, M. M. Glazov, C. Robert, F. Cadiz, G. Wang, T. Taniguchi, K. Watanabe, M. Pierre, W. Escoffier, E. L. Ivchenko, P. Renucci, X. Marie, T. Amand, and B. Urbaszek, Charged Excitons in Monolayer $\mathrm{WSe}_{2}$ : Experiment and Theory, Phys. Rev. B 96, 085302 (2017).

[21] R. A. Suris, V. P. Kochereshko, G. V. Astakhov, D. R. Yakovlev, W. Ossau, J. Nürnberger, W. Faschinger, G. Landwehr, T. Wojtowicz, G. Karczewski, and J. Kossut, Excitons and Trions Modified by Interaction with a Two-Dimensional Electron Gas, Phys. Status Solidi B 227, 343 (2001).

[22] G. Ramon, A. Mann, and E. Cohen, Theory of Neutral and Charged Exciton Scattering with Electrons in Semiconductor Quantum Wells, Phys. Rev. B 67, 045323 (2003).

[23] P. Kossacki, Optical Studies of Charged Excitons in II-VI Semiconductor Quantum Wells, J. Phys. Condens. Matter 15, R471 (2003).

[24] M. T. Portella-Oberli, V. Ciulin, J. H. Berney, B. Deveaud, M. Kutrowski, and T. Wojtowicz, Interacting Many-Body Systems in Quantum Wells: Evidence for Exciton-TrionElectron Correlations, Phys. Rev. B 69, 235311 (2004).

[25] I. Bar-Joseph, Trions in GaAs Quantum Wells, Semicond. Sci. Technol. 20, R29 (2005).

[26] D. Keller, D. R. Yakovlev, G. V. Astakhov, W. Ossau, S. A. Crooker, T. Slobodskyy, A. Waag, G. Schmidt, and L. W. Molenkamp, Magneto-Optics of Two-Dimensional Electron Gases Modified by Strong Coulomb Interactions in ZnSe Quantum Wells, Phys. Rev. B 72, 235306 (2005).

[27] M. Sidler, P. Back, O. Cotlet, A.Srivastava, T. Fink, M. Kroner, E. Demler, and A. Imamoglu, Fermi Polaron-
Polaritons in Charge-Tunable Atomically Thin Semiconductors, Nat. Phys. 13, 255 (2017).

[28] D. K. Efimkin and A. H. MacDonald, Many-Body Theory of Trion Absorption Features in Two-Dimensional Semiconductors, Phys. Rev. B 95, 035417 (2017).

[29] J. Jadczak, A. Delgado, L. Bryja, Y. S. Huang, and P. Hawrylak, Robust High-Temperature Trion Emission in Monolayers of $\mathrm{Mo}\left(\mathrm{S}_{y} \mathrm{Se}_{1-y}\right)_{2}$ Alloys, Phys. Rev. B 95, 195427 (2017).

[30] A. Chernikov, A. M. van der Zande, H. M. Hill, A. F. Rigosi, A. Velauthapillai, J. Hone, and T. F. Heinz, Electrical Tuning of Exciton Binding Energies in Monolayer $\mathrm{WS}_{2}$, Phys. Rev. Lett. 115, 126802 (2015).

[31] F. Cadiz, E. Courtade, C. Robert, G. Wang, Y. Shen, H. Cai, T. Taniguchi, K. Watanabe, H. Carrere, D. Lagarde, M. Manca, T. Amand, P. Renucci, S. Tongay, X. Marie, and B. Urbaszek, Excitonic Linewidth Approaching the Homogeneous Limit in $\mathrm{MoS}_{2}$-Based van der Waals Heterostructures, Phys. Rev. X 7, 021026 (2017).

[32] H. Dery and Y. Song, Polarization Analysis of Excitons in Monolayer and Bilayer Transition-Metal Dichalcogenides, Phys. Rev. B 92, 125431 (2015).

[33] X.-X. Zhang, Y. You, S. Yang, F. Zhao, and T. F. Heinz, Experimental Evidence for Dark Excitons in Monolayer $\mathrm{WSe}_{2}$, Phys. Rev. Lett. 115, 257403 (2015).

[34] F. Wu, F. Qu, and A. H. MacDonald, Exciton Band Structure of Monolayer $\mathrm{MoS}_{2}$, Phys. Rev. B 91, 075310 (2015).

[35] H. Dery, Theory of intervalley Coulomb Interactions in Monolayer Transition-Metal Dichalcogenides, Phys. Rev. B 94, 075421 (2016).

[36] H. Haug and S. Schmitt-Rink, Electron Theory of the Optical Properties of Laser Excited Semiconductors, Prog. Quantum Electron. 9, 3 (1984).

[37] D. Lagarde, L. Bouet, X. Marie, C. R. Zhu, B. L. Liu, T. Amand, P. H. Tan, and B. Urbaszek, Carrier and Polarization Dynamics in Monolayer $\mathrm{MoS}_{2}$, Phys. Rev. Lett. 112, 047401 (2014).

[38] G. Wang, L. Bouet, D. Lagarde, M. Vidal, A. Balocchi, T. Amand, X. Marie, and B. Urbaszek, Valley Dynamics Probed through Charged and Neutral Exciton Emission in Monolayer $\mathrm{WSe}_{2}$, Phys. Rev. B 90, 075413 (2014).

[39] L. Yang, N. A. Sinitsyn, W. Chen, J. Yuan, J. Lou, and S. A. Crooker, Long-Lived Nanosecond Spin Relaxation and Spin Coherence of Electrons in Monolayer $\mathrm{MoS}_{2}$ and $\mathrm{WS}_{2}$, Nat. Phys. 11, 830 (2015).

[40] X. Song, S. Xie, K. Kang, J. Park, and V. Sih, Long-Lived Hole Spin/Valley Polarization Probed by Kerr Rotation in Monolayer $\mathrm{WSe}_{2}$, Nano Lett. 16, 5010 (2016).

[41] D. Bohm and D. Pines, A Collective Description of Electron Interactions: III. Coulomb Interactions in a Degenerate Electron Gas, Phys. Rev. 92, 609 (1953).

[42] A. Bardasis and J. R. Schrieffer, Excitons and Plasmons in Superconductors, Phys. Rev. 121, 1050 (1961).

[43] Z. Sun, D. N. Basov, and M. M. Fogler, Adiabatic Amplification of Plasmons and Demons in 2D Systems, Phys. Rev. Lett. 117, 076805 (2016).

[44] D. Pines, Collective Energy Losses in Solids, Rev. Mod. Phys. 28, 184 (1956).

[45] L. V. Keldysh, Coulomb Interaction in Thin Semiconductor and Semimetal Films, JETP Lett. 29, 658 (1979). 
[46] P. Cudazzo, I. V. Tokatly, and A. Rubio, Dielectric Screening in Two-Dimensional Insulators: Implications for Excitonic and Impurity States in Graphene, Phys. Rev. B 84, 085406 (2011).

[47] R. Schmidt, G. Berghauser, R. Schneider, M. Selig, P. Tonndorf, E. Malic, A. Knorr, S. Michaelis de Vasconcellos, and R. Bratschitsch, Ultrafast Coulomb-Induced Intervalley Coupling in Atomically Thin $\mathrm{WS}_{2}$, Nano Lett. 16, 2945 (2016).

[48] S. L. Adler, Quantum Theory of the Dielectric Constant in Real Solids, Phys. Rev. 126, 413 (1962).

[49] T. Tudorovskiy and S. A. Mikhailov, Intervalley Plasmons in Graphene, Phys. Rev. B 82, 073411 (2010).

[50] M. Danovich, V. Zolyomi, and V. I. Fal'ko, Dark Trions and Biexcitons in $\mathrm{WS}_{2}$ and $\mathrm{WSe}_{2}$ Made Bright by e-e Scattering, Sci. Rep. 7, 45998 (2017).

[51] G. D. Mahan, Many-Particle Physics, 3rd ed. (Kluwer Academics, New York, 2000).

[52] H. J. Vidberg and J. W. Serene, Solving the Eliashberg Equations by Means of N-point Pade Approximants, J. Low Temp. Phys. 29, 179 (1977).

[53] K. Shindo, Effective Electron-Hole Interaction in Shallow Excitons, J. Phys. Soc. Jpn. 29, 287 (1970).

[54] H. Haug and S. W. Koch, Quantum Theory of the Optical and Electronic Properties of Semiconductors, 3rd ed. (World Scientific, Singapore, 1994).

[55] S. Schmitt-Rink, C. Ell, and H. Haug, Many-Body Effects in the Absorption, Gain, and Luminescence Spectra of Semiconductor Quantum-Well Structures, Phys. Rev. B 33, 1183 (1986).

[56] A. Kormànyos, G. Burkard, M. Gmitra, J. Fabian, V. Zòlyomi, N. D. Drummond, and V. I. Fal'ko, k · p Theory for Two-Dimensional Transition Metal Dichalcogenide Semiconductors, 2D Mater. 2, 049501 (2015).

[57] K. Kim, S. Larentis, B. Fallahazad, K. Lee, J. Xue, D. C. Dillen, C. M. Corbet, and E. Tutuc, Band Alignment in $\mathrm{WSe}_{2}$-Graphene Heterostructures, ACS Nano 9, 4527 (2015).

[58] A. Esser, R. Zimmermann, and E. Runge, Theory of Trion Spectra in Semiconductor Nanostructures, Phys. Status Solidi B 227, 317 (2001).

[59] M. M. Ugeda, A. J. Bradley, S.-F. Shi, F. H. da Jornada, Y. Zhang, D. Y. Qiu, W. Ruan, S.-K. Mo, Z. Hussain, Z.-X. Shen, F. Wang, S. G. Louie, and M. F. Crommie, Giant Bandgap Renormalization and Excitonic Effects in a Monolayer Transition Metal Dichalcogenide Semiconductor, Nat. Mater. 13, 1091 (2014).

[60] A. Chernikov, C. Ruppert, H. M. Hill, A. F. Rigosi, and T. F. Heinz, Population Inversion and Giant Bandgap Renormalization in Atomically Thin $\mathrm{WS}_{2}$ Layers, Nat. Photonics 9, 466 (2015).

[61] X.-X. Zhang, T. Cao, Z. Lu, Y.-C. Lin, F. Zhang, Y. Wang, Z. Li, J. C. Hone, J. A. Robinson, D. Smirnov, S. G. Louie, and T. F. Heinz, Nat. Nanotechnol. 12, 883 (2017).

[62] Ž. Osolin and R. Žitko, Padé Approximant Approach for Obtaining Finite-Temperature Spectral Functions of Quantum Impurity Models Using the Numerical Renormalization Group Technique, Phys. Rev. B 87, 245135 (2013).
[63] T. C. Berkelbach, M. S. Hybertsen, and D. R. Reichman, Theory of Neutral and Charged Excitons in Monolayer Transition Metal Dichalcogenides, Phys. Rev. B 88, 045318 (2013).

[64] A. Chernikov, T. C. Berkelbach, H. M. Hill, A. Rigosi, Y. Li, O. B. Aslan, D. R. Reichman, M. S. Hybertsen, and T. F. Heinz, Exciton Binding Energy and Nonhydrogenic Rydberg Series in Monolayer $\mathrm{WS}_{2}$, Phys. Rev. Lett. 113, 076802 (2014).

[65] D. K. Zhang, D. W. Kidd, and K. Varga, Excited Biexcitons in Transition Metal Dichalcogenides, Nano Lett. 15, 7002 (2015).

[66] A. Thilagam, Exciton Complexes in Low Dimensional Transition Metal Dichalcogenides, J. Appl. Phys. 116, 053523 (2014).

[67] B. Ganchev, N. Drummond, I. Aleiner, and V. Fal'ko, Three-Particle Complexes in Two-Dimensional Semiconductors, Phys. Rev. Lett. 114, 107401 (2015).

[68] G. A. Narvaez, P. Hawrylak, and J. A. Brum, The Role of Finite Hole Mass in the Negatively Charged Exciton in Two Dimensions, Physica E (Amsterdam) 9, 716 (2001).

[69] M. Z. Mayers, T. C. Berkelbach, M. S. Hybertsen, and D. R. Reichman, Binding Energies and Spatial Structures of Small Carrier Complexes in Monolayer TransitionMetal Dichalcogenides via Diffusion Monte Carlo, Phys. Rev. B 92, 161404 (2015).

[70] M. Van der Donck, M. Zarenia, and F. M. Peeters, Excitons and Trions in Monolayer Transition Metal Dichalcogenides: A Comparative Study between the Multiband Model and the Quadratic Single-Band Model, Phys. Rev. B 96, 035131 (2017).

[71] P. Hawrylak, Optical Properties of a Two-Dimensional Electron Gas: Evolution of Spectra from Excitons to Fermi-Edge Singularities, Phys. Rev. B 44, 3821 (1991).

[72] M. Lax and J. J. Hopfield, Selection Rules Connecting Different Points in the Brillouin Zone, Phys. Rev. 124, 115 (1961).

[73] P. Li and H. Dery, Theory of Spin-Dependent PhononAssisted Optical Transitions in Silicon, Phys. Rev. Lett. 105, 037204 (2010).

[74] P. Li, D. Trivedi, and H. Dery, Spin-Dependent Optical Properties in Strained Silicon and Germanium, Phys. Rev. B 87, 115203 (2013).

[75] S. Hausser, G. Fuchs, A. Hangleiter, and K. Streubel, Auger Recombination in Bulk and Quantum Well InGaAs, Appl. Phys. Lett. 56, 913 (1990).

[76] A. S. Polkovnikov and G. G. Zegrya, Auger Recombination in Semiconductor Quantum Wells, Phys. Rev. B 58, 4039 (1998).

[77] M. Danovich, V. Zólyomi, V. I. Fal'ko, and I. L Aleiner, Auger Recombination of Dark Excitons in $\mathrm{WS}_{2}$ and $\mathrm{WSe}_{2}$ Monolayers, 2D Mater. 3, 035011 (2016).

[78] I. Kylänpää and H.-P. Komsa, Binding Energies of Exciton Complexes in Transition Metal Dichalcogenide Monolayers and Effect of Dielectric Environment, Phys. Rev. B 92, 205418 (2015).

[79] Y. You, X.-X. Zhang, T. C. Berkelbach, M. S. Hybertsen, D. R. Reichman, and T. F. Heinz, Observation of Biexcitons in Monolayer $\mathrm{WSe}_{2}$, Nat. Phys. 11, 477 (2015). 
[80] K. A. Velizhanin and A. Saxena, Excitonic Effects in 2D Semiconductors: Path Integral Monte Carlo Approach, Phys. Rev. B 92, 195305 (2015).

[81] R. Ya. Kezerashvili and S. M. Tsiklauri, Trion and Biexciton in Monolayer Transition Metal Dichalcogenides, Few-Body Syst. 58, 18 (2017).

[82] M. Szyniszewski, E. Mostaani, N. D. Drummond, and V. I. Fal'ko, Binding Energies of Trions and Biexcitons in Two-Dimensional Semiconductors from Diffusion Quantum Monte Carlo Calculations, Phys. Rev. B 95, 081301(R) (2017).

[83] B. Scharf, G. Xu, A Matos-Abiague, and I. Žutić, Magnetic Proximity Effects in Transition-Metal Dichalcogenides: Converting Excitons, Phys. Rev. Lett. 119, 127403 (2017).

[84] Y. Ye, J. Xiao, H. Wang, Z. Ye, H. Zhu, M. Zhao, Y. Wang, J. Zhao, X. Yin, and X. Zhang, Electrical Generation and Control of the Valley Carriers in a Monolayer Transition Metal Dichalcogenide, Nat. Nanotechnol. 11, 598 (2016).

[85] C. Zhao, T. Norden, P. Zhao, Y. Cheng, P. Zhang, F. Sun, P. Taheri, J. Wang, Y. Yang, T. Scrace, K. Kang, S. Yang, G. Miao, R. Sabirianov, G. Kioseoglou, A. Petrou, and H. Zeng, Enhanced Valley Splitting in Monolayer $\mathrm{WSe}_{2}$ Due to Magnetic Exchange Field, Nat. Nanotechnol. 12, 757 (2017).

[86] D. Zhong, K. L. Seyler, X. Linpeng, R. Cheng, N. Sivadas, B. Huang, E. Schmidgall, T. Taniguchi, K. Watanabe, M. A. McGuire, W. Yao, D. Xiao, K.-M. C. Fu, and X. Xu, Van der Waals Engineering of Ferromagnetic Semiconductor Heterostructures for Spin and Valleytronics, Sci. Adv. 3, e1603113 (2017).

[87] D. Pines, Electron Interaction in Solids, Can. J. Phys. 34, 1379 (1956).

[88] V. Radhakrishnan, Superconductivity in Transition Elements, Phys. Lett. 16, 247 (1965).

[89] E. A. Pashitskii, Plasmon Mechanism of Superconductivity in Degenerate Semiconductors and Semimetals, Sov. Phys. JETP 28, 1267 (1969).

[90] J. Ruvalds, Plasmons and High-Temperature Superconductivity in Alloys of Copper Oxides, Phys. Rev. B 35, 8869(R) (1987).
[91] V. M. Krasnov, S.-O. Katterwe, and A. Rydh, Signatures of the Electronic Nature of Pairing in High-T $T_{c}$ Superconductors Obtained by Non-equilibrium Boson Spectroscopy, Nat. Commun. 4, 2970 (2013).

[92] B. N. Ganguly and R. F. Wood, Acoustical Plasmons, Phonon Anomalies, and Superconductivity in TransitionMetal Systems, Phys. Rev. Lett. 28, 681 (1972).

[93] E. A. Pashitskii, Plasmon Mechanism of High-Temperature Superconductivity in Cuprate Metal-Oxide Compounds, Sov. Phys. JETP 76, 425 (1993).

[94] Y. Takada, Plasmon Mechanisms of Superconductivity in Two- and Three-Dimensional Electron Gas, J. Phys. Soc. Jpn. 45, 786 (1978).

[95] J. Ruhman and P. A. Lee, Superconductivity at Very Low Density: The Case of Strontium Titanate, Phys. Rev. B 94, 224515 (2016).

[96] G. Vignale and K. S. Singwi, Possibility of Superconductivity in Electron-Hole Liquid, J. Phys. Soc. Jpn. 45, 786 (1978).

[97] R. Blank and H. Haug, Theory of Plasmon-Assisted Electron Pairing in Semiconductors, Phys. Status Solidi B 188, 105 (1995).

[98] M. Z. Maialle, E. A. de Andrada e Silva, and L. J. Sham, Exciton Spin Dynamics in Quantum Wells, Phys. Rev. B 47, 15776 (1993).

[99] H. Yu, G.-B. Liu, P. Gong, X. Xu, and W. Yao, Dirac Cones and Dirac Saddle Points of Bright Excitons in Monolayer Transition Metal Dichalcogenides, Nat. Commun. 5, 3876 (2014).

[100] J. P. Echeverry, B. Urbaszek, T. Amand, X. Marie, and I. C. Gerber, Splitting between Bright and Dark Excitons in Transition Metal Dichalcogenide Monolayers, Phys. Rev. B 93, 121107 (2016).

[101] R. Geick, C. H. Perry, and G. Rupprecht, Normal Modes in Hexagonal Boron Nitride, Phys. Rev. 146, 543 (1966).

[102] T. Sohier, M. Calandra, and F. Mauri, Two-Dimensional Fröhlich Interaction in Transition-Metal Dichalcogenide Monolayers: Theoretical Modeling and First-Principles Calculations, Phys. Rev. B 94, 085415 (2016). 